\title{
Operationalizing Goal Directedness: An Empirical Route to Advancing a Philosophical Discussion
}

\author{
Jong Gwan Lee ${ }^{* \dagger}$ and Daniel W. McShea*ł
}

Goal directedness is one of the most commonly observed behavior patterns in biology, exemplified by systems ranging in complexity from cellular migration to human motivations. Philosophers have long tried to understand goal directedness in terms of necessary and sufficient conditions, but no consensus has been reached. Here we take an entirely novel approach to goal directedness, postponing the search for necessary and sufficient conditions, and instead trying to advance understanding by an empirical route. In particular, we introduce quantitative measures of goal directedness, applicable to systems that are generally agreed to be goal directed. The measures allow one to assess two signature properties of goal-directed systems, persistence and plasticity. Persistence is the tendency for an entity that is on a trajectory toward a goal to return to that trajectory following perturbations. Plasticity we understand as the tendency for an entity to find a trajectory toward a goal from a variety of different starting distances. We demonstrate the metrics by applying them to goal-directed behavior in two biological systems, bacteria moving up a chemoattractant gradient and a human following a heat gradient. Our approach reveals goal directedness to be an empirically tractable notion, one that makes possible a variety of comparative studies in biology, including comparing degree of goal directedness in different species, or in one species under different conditions, as well as studying evolutionary trends. More generally, the metrics make it possible to investigate the correlates and causes of goal-directed behavior. Finally, our approach challenges the conventional view of goal directedness as a discrete and unitary property, by showing that it can be treated as continuous, as a matter of degree, and that it can be broken down into at least two, and possibly more, partly independent components.

$$
\begin{gathered}
\text { Keywords } \\
\text { goal directedness } \bullet \text { teleology } \bullet \text { Nagel, Ernest } \bullet \text { persistence } \bullet \text { plasticity }
\end{gathered}
$$

*Department of Biology, Duke University, Box 90338, Durham, NC 27708, USA

$\dagger$ Current Affiliation: Program in Biological and Biomedical Sciences, Harvard Medical School,

Boston, MA 02115, USA, jonggwanlee@g.harvard.edu

‡ Corresponding Author, dmcshea@duke.edu

Received 2 August 2019; Revised 24 December 2019; Accepted 13 January 2020 doi:10.3998/ptpbio.16039257.0012.005

๑ OPEN ACCESS - PTPBIO.ORG 


\section{Introduction}

Two signature features of goal-directed systems are persistence and plasticity (Nagel 1979, Sommerhoff 1950). Persistence is the tendency for an entity on some trajectory to return to that trajectory following perturbations. For example, a homing torpedo that is attracted off course by the sounds of a passing pod of whales returns to a trajectory toward a target ship when the distraction disappears. In its pursuit of the goal, the target ship, it "persists." A bacterium moving toward a piece of sugar at the center of the petri dish persists toward that goal despite the small random currents within the fluid that repeatedly deflect it from that trajectory. A student walking to class stops to say hello to a friend but then returns to a trajectory toward the lecture hall. She persists.

Plasticity has been understood as the tendency for an entity to find a particular trajectory from a variety of different starting points. A homing torpedo can be launched from south or west of the target ship and yet still find a trajectory toward the ship. The bacterium finds a trajectory toward the food whether it starts at the edge of the petri dish or near the center. The student moves toward the lecture hall whether she starts from the dining hall or her dorm. The behavior of all of these entities is plastic.

The homing torpedo, the food-seeking bacterium, and the student are all goal directed. And persistence and plasticity are properties of the trajectories that move them toward their goals. This paper develops two metrics, a measure of persistence and a measure of plasticity, which together operationalize the concept of goal directedness. Our hope is that they will be useful for the empirical study of goal directedness in biology and other fields. But their main purpose is not scientific. It is to raise conceptual issues and to advance the discussion of goal directedness in the philosophy literature. In particular, we argue that an empirical approach, and even empirical results, point to some course corrections in the philosophical discussion. They suggest that goal-directedness should be treated as a matter of degree, rather than all-or-none, and as multidimensional or composite, rather than unitary or unalloyed. Our approach also offers an alternative to the perhaps-premature search for necessary and sufficient conditions.

\section{Background}

There have been two major lines of thought in the philosophical analysis of goal directedness, behavioral and mechanistic (Garson 2016). Sommerhoff and Braithwaite were two of the earliest philosophers who took the behavioral approach. Sommerhoff (1950) argued that in order for a system to be goal directed, it must have some sort of behavioral flexibility, modifying its pattern of behavior with respect to its target to attain its goal. Meanwhile, Braithwaite (1953) suggested plasticity, i.e. movement towards the goal under a variety of circumstances, as a distinguishing criterion for goal directedness.

On the other hand, Rosenblueth et al. (1943) made one of the first attempts to identify a goal-directed behavior in terms of the internal mechanism of the behaving object. They argued that all goal-directed behaviors must be governed by negative feedback mechanisms, which uses the signals from the goal to modify and direct the behavior of the goal-directed entity. Nagel (1979) further developed the mechanistic approach, incorporating persistence and plasticity in his system-property view. In his account of goal directedness, he dissected a system into a number of parts. In a goal-directed system, these parts respond to changes in each other and behave in coordinated manner to attain the goal. In other words, when a deviation causes some parts to change, other parts respond subsequently to this change in a way that corrects for the deviation and the goal is attained (Nagel 1979; see also Ehring 1984). In this way, Nagel argued that goal directedness arises by virtue of a system's internal organization. In his view, a homing 
torpedo is goal directed because, when a movement of the target ship causes the angle of the sound field to change, the subsequent, coordinated interactions among multiple parts occur to turn the rudder of the torpedo and to restore the proper trajectory toward the ship in a new location. In contrast, a ball rolling around in a bowl looks goal directed because it consistently returns to a trajectory toward the bottom of the bowl, but it is not, according to Nagel, because it does not have an internal mechanism that involves multiple parts that respond to each other.

Mayr (1974) suggested the notion of teleonomy for understanding goal-directed systems. A teleonomic entity is one that persists towards a goal using some sort of internal program. And he distinguished teleomatic behaviors from teleonomic ones, based on differences in underlying mechanism. If a goal-directed behavior is governed only by natural laws, as in the case of the ball in the bowl, then it is "teleomatic," whereas "teleonomic" behaviors are governed by complex internal programs.

The mission of these philosophical accounts has always been to define goal directedness, to discover necessary and sufficient conditions for it. But so far, no consensus has been achieved. One recurring criticism is the problem of "overbreadth," definitions that encompass too many systems, including those that exhibit persistence and plasticity but that we are not inclined to call goal directed (Garson 2016; see also Woodfield 1976), like the ball in the bowl. The mechanistic tradition largely avoided the problem of overbreadth, but it encountered other issues. For instance, Scheffler (1959) proposed the problem of "missing goal-object," that raises problems for negative feedback as a central criterion. He argued that people can be directed by a nonexistent, imaginary goal, such as the philosopher's stone and the Holy Grail, even though there is not, and cannot be, any feedback signal from the goal that could modify their trajectories. And Ehring (1984) raised problems with Nagel's definition of goal directedness. One is that his view cannot handle impossible goals, because Nagel required that a system have the capacity to adjust its parameters, to find the right combination of variables, to achieve a goal (Ehring 1984).

Recently, one of us (DWM) took a different and more pragmatic approach to goal directedness (McShea 2012, 2016a,b). Instead of searching for necessary and sufficient conditions, for the defining qualities of goal directedness, he instead sought to characterize goal directed systems in terms of their structure. The mission was to identify a common structure or organization present in at least the vast majority of goal directed systems. He proposed that all goal directed systems have a hierarchical structure in which a goal-directed entity moves within a directing "field." This field concept represents the totality of the teleologically relevant conditions, that is, the environmental factors that affect the goal-directed behavior of an entity. For instance, a bacterium swimming toward the food source on a petri dish is contained within a field, the chemical concentration gradient of the food. For a person who is groping along the wall for a light switch in a dark room, the topography of the wall is the field. The field is the set of external conditions that allows the entity to behave in a goal-directed manner, persistently and plastically. When they deviate from the proper trajectory toward the goal, the field directs them back. And the field makes it possible for the entities contained in it to find the trajectory toward the goal from various starting points. In other words, the field is a necessary structural component for goal-directed behaviors. The approach is inspired by Sommerhoff's and Nagel's, in that it focuses on persistence and plasticity, but the mission is to understand goal directedness in engineering terms, to find commonalities in the way that persistence and plasticity are achieved in most (if not all) biological systems, rather than to find necessary and sufficient conditions.

The present study adopts McShea's engineering approach, but the usefulness of the metrics developed does not depend on his field theory. The metrics are applicable to any scheme for understanding goal directedness that recognizes persistence and plasticity as signature behaviors. 


\section{Mission}

As mentioned above, the narrow mission here is to provide tools for the study of goal-directed behavior in biology. The metrics we develop can be used to compare goal-directed behaviors among individuals of the same species or among species, potentially allowing us to say for example whether one species is more persistent or plastic than another in certain tasks. They can also be used to investigate causes and correlates of goal-directed behavior, to discover for example whether changes in some environmental condition affect persistence and plasticity. Finally, they can be used to test for trends toward increased (or decreased) goal directedness in evolution. The tools will help to address almost any question related to goal directedness that requires measurement.

Also our metrics offer a unified approach to the empirical study of goal directedness. Measures and theoretical frameworks have been developed in various other contexts, as in the study of chemotaxis (e.g., McCutcheon 1946; Orr and Ward 1978; Beauvais et al. 1995; Lind et al. 1995; Tanaka et al. 2019; Ramirez-Gomez et al. 2019; Varennes et al. 2019), in the study of homeostasis (e.g., Gershenson and Fernandez 2012; Atkin et al. 2005), and in movement ecology (e.g., Marsh and Jones 1988; Benhamou and Bovet 1992; Turchin 1998; Benhamou 2006; Nathan et al. 2008). In these areas, goal directedness has been an implicit component of the conversation, but none of the measures have been developed expressly for studying goal directedness in general. Our focus is explicitly on goal directedness and comes out of the discussion of it in philosophy.

Our broader mission has a number of dimensions:

1. The study of goal directedness in the philosophy of biology has been missing an empirical dimension. The focus has been on the words and concepts, without a clear understanding of the phenomenon itself. One route to understanding is empirical studies of real goaldirected systems, and thus here we try to nudge the discussion of goal-directedness toward an empirical approach by demonstrating the feasibility of operationalizing it, by showing that a generally applicable measure can be used in the analysis of data in disparate goaldirected systems. We demonstrate this by doing it.

2. For the most part, the twentieth century discussion of goal directedness in philosophy treated the concept as dichotomous. For a given system, its behavior was considered to be either goal directed or not. Our findings here suggest a continuum. At the same time, it dramatizes the sense in which degree of goal directedness is a function of context. An entity can have some degree of goal directedness in some circumstances, and either more or less in others.

3. The past discussion has for the most part treated goal directedness as a single process, as one dimensional. For example, Nagel recognized both persistence and plasticity as necessary for goal directness but treats goal directedness itself as a unitary phenomenon. Here we show not only that it could have at least two components, but that they seem to be partly independent of each other. Thus, like intelligence, goal directedness could be composite, multidimensional. In other words, the conventional ontology could be wrong. Goal directedness could be two (or more) concepts, not one.

4. The philosophical debate has been mainly interested in the problem of finding necessary and sufficient conditions for goal directedness. As discussed, this approach has been unsuccessful so far in that no consensus has been achieved on what those necessary and sufficient conditions are. Our approach opens up a new front, so to speak. By showing 
how the problem can be attacked empirically, it moves the project of understanding goal directedness into discovery mode. The hope is that by learning more about how goal directedness arises in various systems and how it changes under various conditions and in evolution, we will be better positioned to talk about what goal directedness is.

5. Finally, in our most ambitious moments, we imagine ourselves contributing to a revitalization of the now-languishing study of goal directedness in philosophy.

In the first section below, we set the stage, explaining our general approach to measurement and giving some important caveats. The next three sections present the metrics, and the two sections after that show how they can be applied to two biological goal-directed systems, Escherichia coli chemotaxis and a human performing a purposeful task. Finally, the discussion will consider the consequences for our understanding of goal directedness. An appendix offers step-by-step instructions for calculating persistence and plasticity, and explains in detail how the values for the $E$. coli and the human trajectories were generated.

\section{Measuring Persistence and Plasticity}

Persistence is operationalized here as how much more likely, on average, an entity is to move toward the goal, rather than away from it, compared to chance alone, following a perturbation. That is, persistence is bias with respect to the goal. Thus when the sound of a passing pod of whales deflects a homing torpedo from a trajectory toward a target ship, the torpedo finds itself in some location, and persistence is the bias toward the target ship, the tendency of the torpedo to move in the direction of the target ship from that new location. Notice first that the nature and even the effect of the perturbation itself does not matter here. So that in principle, persistence can also be understood simply as the tendency to move toward the goal from any arbitrary point. Notice too that a correction for chance movement is necessary. An entity that is not goal directed, that is moving randomly, will by chance alone move toward the goal sometimes, and in our understanding, these chance movements do not count toward persistence. Other factors that direct an entity, including certain boundary conditions, discussed later, also require a correction factor. For now, we assume that the entity moves in an unbounded space, so that movements are possible in all directions.

Plasticity is the effect of starting location on ability to find a goal, which here we operationalize as the degree to which, on average, an increase in starting distance from the goal increases trajectory length. The Nagel (1979) and Sommerhoff (1950) understanding of plasticity is somewhat different, in that they ask whether the goal can be attained from a variety of starting points, making a distinction between entities that are plastic and those that are not. However, at least in the one and two dimensional cases considered here, all entities, goal directed or not, even if they are following a random walk, will eventually reach the goal (Doyle and Snell 1984), unless there are boundaries or repelling forces. Given this, a more meaningful question to ask is about the effect of starting distance on trajectory length. A similar approach that uses the ratio of the cell displacement toward the gradient to the total migration distance has been taken in the literature on chemotaxis, to calculate a "chemotaxis index" (e.g., McCutcheon 1946; Qasaimeh et al. 2018).

For plasticity, the comparison is not with the behavior of a random entity but with a perfectly goal directed one. Entities that start further away from the goal are expected to take longer to get there, even if they are perfectly goal directed and make a beeline for the goal. So plasticity is how much longer an entity's trajectory is compared to such a perfectly goal-directed entity. 
Those that take much longer, given the starting distance, are less plastic, and in that sense less goal directed.

These metrics are tailored to goal-directed systems in which the goal is external to the entity, relatively stable in space and time, and attractive to (as opposed to repelling of) the goal-directed entity. A further assumption is that the structure or organization of the goal-directed entity is also stable so that its goal-directed propensities do not change over time. Also, our examples are all in physical spaces, although as we discuss later, the metrics will work for entities moving in any kind of state space. For example, a thermostat-driven heating and cooling system makes the behavior of the temperature of a house goal directed in a state space defined by temperature. The goal-directed thought processes of a person trying to solve a problem in his or her head are "moving" in a state space defined by the problem at hand.

The major virtue of these metrics is that they allow us to quantitatively evaluate the behavior of similar-or in some cases, quite disparate - goal-directed entities, to measure their behavior in the same terms. The metrics can be applied to generate numeric values for a wide range of goaldirected behaviors, from tropisms in simple organisms (e.g., bacteria moving up a gradient), to elaborate innate goal-directed behaviors in more complex organisms (e.g., migrating birds), to the more flexible purposeful behavior patterns in humans and other vertebrates, to goal-directed behaviors in human artifacts (e.g., AI-driven robots).

\section{Clarifications}

The metrics do not provide a standard that can by itself define or identify a goal-directed system. In other words, resultant values that represent degrees of persistence and plasticity will not by themselves allow the user to decide whether it is appropriate or not to call a system goal directed. It is not our mission to identify any numerical cut-off points, values of persistence and plasticity above which a system should be called goal directed and below which it should not. In other words, the project is not, as we have said, to find necessary and sufficient conditions.

Another caveat is that the notions of persistence and plasticity in this paper are specifically drawn from and pertinent to the philosophical literature on goal directedness. For instance, the notion of persistence used in this paper is different from that in the literature on random walks. In the random walk literature, persistence refers to the tendency for an entity to move in the same direction as its previous movement (Patlak 1953). In other words, persistence indicates a directional correlation between successive moves (Codling et al. 2008). In contrast, here-as in the literature on goal directedness-persistence is a function of movements toward or away from the goal, and the relationships among successive steps are not relevant. For instance, a bacterium continuously swimming away from the food source in a straight line would have high persistence in the sense in which the term is used in the random walk literature. However, in our scheme, the same bacterium would not be persistent at all. Likewise, the notion of plasticity used in this paper is different than in other contexts, such as developmental plasticity in biology (West-Eberhard 2003) or plasticity in physics (Hill 1950).

Third, the metrics are statistical in the sense that they assess goal directedness not based on a single trajectory of a single entity, but using data on the movements of a population of entities or multiple trajectories of the same entity.

Finally, we do not claim that the metrics developed here are the only possible route to measuring persistence and plasticity, nor are persistence and plasticity the only possible way to conceptualize goal-directed behavior. Many other approaches are possible, and we discuss some of them in the next subsection. 


\section{Adjacent Literatures}

Our approach to the concept of goal directedness, as well as the interpretations of persistence and plasticity proposed above for operationalization, is in line with some of the established methods in various fields studying biological movements. A variety of approaches, involving diverse random walk models and statistical analyses, have been developed to evaluate directional bias and navigation ability in biological systems (Bovet and Benhamou 1988; Turchin 1998; Benhamou 2006; Codling et al. 2008). For instance, one classic example of such an approach was demonstrated by Kareiva and Shigesada (1983), in which the mean squared displacement of butterflies under different ecological circumstances (ovipositing versus nectar-feeding) was evaluated with respect to the expected pattern of a null model. Hill and Häder (1997) analyzed swimming trajectories of algae guided by negative gravitaxis and phototaxis for parameters such as swimming speed and angular distribution, to quantitatively characterize the goal-directed behavior of algae. Similarly, Schultz and Crone (2001) analyzed flight trajectories of butterflies both within and outside of their usual habitat (where directional bias is not expected) and at the edge of their habitat (where directional bias is expected) to quantify the bias component (the tendency to move toward the habitat) with respect to the correlated component (the tendency to continue the same direction). And Turchin (1998) introduced a way to evaluate directional bias of caterpillars, which compares probabilities of making turns toward or away from an attractor as a function of distance from the attractor. Thus, detecting and quantifying directional bias in organismal movement has been a long-standing project in the biological literature (e.g., Marsh and Jones 1988; Bailey et al. 2018; Bearon and Durham 2019; Peleg and Mahadevan 2016; McClintock et al. 2012; Bailey and Thompson 2006; Fortin et al. 2005; Papastamatiou et al. 2011; Khaldy et al. 2019).

Although called plasticity here for consistency with the philosophical literature, similar aspects of biological movements have been studied under such names as chemotaxis index (e.g., McCutcheon 1946; Qasaimeh et al. 2018; Varennes et al. 2019), and straightness index or navigation efficiency (Batschelet 1981; Benhamou and Bovet 1992; Benhamou 2004; Codling et al. 2008; Bailey et al. 2018; Benhamou et al. 2003; Weimerskirch et al. 2002), commonly measured as the ratio of net displacement to the total trajectory length. Often, such measures are employed to measure the tortuosity or convolutedness of movement path, which are thought to be key parameters in animal behaviors such as dispersal, foraging, and orientation (Benhamou 2004). In addition, approaches such as fractal analysis and sinuosity index have been applied to measure tortuosity and, more generally, to characterize movement paths (Bovet and Benhamou 1988; Turchin 1996; Benhamou 2004).

Thus, there is substantial overlap between established approaches to and measures of biological movements and ours. However, to our knowledge, these aspects of biological movementalthough central to the literature in a variety of fields-have not been treated under the heading of the goal directedness, nor have any connections been drawn between them and the literature on goal directedness in the philosophy of biology. We take those two conceptual steps here.

\section{Persistence and Plasticity on a Real Number Line}

Imagine a one-dimensional space, such as a real number line, where zero is the goal. At any given time, the entity occupies a point corresponding to some integer and moves one unit distance in each time interval to an adjacent integer. When the entity arrives at zero, it stops moving, and it is "the end of the game." In effect, the goal is an absorbing boundary. 
Suppose that the entity behaves in a perfectly goal-directed way. It moves towards the goal without any deviation, running straight toward 0 from its starting point. Its persistence is maximal. Also, the trajectory length of the entity to reach the goal will always be equal to the starting distance, every step reducing the distance between the goal and the goal-directed entity. So its plasticity is also maximal.

Now suppose that an entity is unbiased, behaving in a completely random, non-goal-directed way, with an equal chance of moving right or left, regardless of its location relative to the goal. In other words, at any time, the entity has a 50\% chance of moving toward the goal as well as away from the goal. Persistence is minimal. As mentioned above, random moves toward the goal do not count toward persistence. Persistence is a tendency, so that to compute it, random movements toward the goal need to be factored out. (See below.)

As noted above, for plasticity we do not use randomness as a reference condition. It is true that for a random, non-goal-directed entity, starting at even a moderate distance from the goal, the average trajectory length will be far larger than the starting distance. So plasticity would be very low. However, for a random entity, trajectory lengths vary enormously over a number of trials. That is, random walks show no stable relationship between starting distance and trajectory length (Berg 1993). Thus, for plasticity, although trajectory length increases with starting distance in the random-walk case, it is not meaningful to talk about the amount of increase. And for that reason, plasticity is based not on a comparison with the random-walk case, but with the perfectly goal-directed case. (See below.)

The behavior of goal-directed entities in the two dimensional case is not qualitatively different, and in fact, the measures we develop below are applicable to both.

\section{Quasi-independence}

The fact that persistence and plasticity are both high for a perfectly goal-directed entity and both low for a randomly moving entity, naturally raises the question of whether they are independent, whether they are actually measuring different things. A simple thought experiment demonstrates that they are at least somewhat independent. Consider two people pursuing the same goal, one doing so with high persistence and low plasticity and the other with the reverse. The first person always has the goal in his mind and works toward it but does so poorly and inefficiently. If his behavior were represented as a trajectory, it would be a series of fine zigzags, the vast majority of which reduce the distance between the goal (high persistence), but only slightly, producing a very long trajectory (low plasticity). In contrast, the second person does not think much about the goal and most of the time makes only small movements, all random with respect to it (low persistence). But on the rare occasion when she does focus on the goal, she is highly directed and efficient, making great progress toward it (high plasticity). As a trajectory, this would appear as long series of small moves at right angles to the goal, or even occasionally away from it, with rare bursts of perfect, highly-directed moves straight toward it. Independence is revealed more concretely by the demonstrations below.

\section{Persistence}

A simple measure of persistence would be: $P=\frac{G}{N}$, where $P$ is persistence, $G$ is the number of moves the entity makes toward the goal (hereafter, "good moves") in some time interval, and $N$ is the total number of moves made in the same time interval. However, even in an entity with no goal directed capability at all, some of its moves will be toward the goal simply by chance. Thus, the metric will factor out the movement toward the goal that is expected for an entity that 
is behaving randomly. This leads to: $P=2 \frac{G}{N}-1$. (Negative values are possible in real data, but are never the expectation for an entity with a bias toward the goal, and would be interpretable as randomness.)

\section{Teleologically Relevant Conditions}

The factors that affect the movement of a goal-directed entity, causing its trajectory to deviate from randomness, are what we call teleologically relevant conditions. The goal itself-along with the substances or fields emanating from it that allow an entity to pursue it - is a teleologically relevant condition. But there is another category of factors, those arising from non-goal sources, and in real systems some of these will affect the trajectory of the goal-directed entity, potentially confounding our measure of persistence. So for example, if a bacterium moving up a food gradient is affected by a local magnetic field, as well as by the food gradient, then the magnetic field is a teleologically relevant condition, and-if the measurer is interested in the goal-directed behavior of the bacterium in response to the food gradient - the effect of the magnetic field needs to be factored out in the computation of persistence. In a simple case, where the field acts the same everywhere, drawing the entity away from the goal and decreasing the probability of moving toward the goal, factoring it out might involve simply adding a correction factor to the equation above. We do that in the next section.

However, where the fields and its effects vary in space, the situation is more complicated. Consider a bacterium positioned near the edge of a petri dish with a food source at the center. The bacterium will tend to move toward the goal whether it is attracted to the food source or not, simply because the edge of the dish limits its options. And persistence calculated using the equation above will exaggerate the goal-directed persistence of the bacterium. To accommodate this confounding teleologically relevant condition, we need a way to factor out the effect of the edge. For instance, a measurer can subdivide the environment and calculate a separate persistence value for each subdivision, one for bacteria near the edge and another for those away from the edge.

Thus, with these refinements, persistence can be understood as bias toward the goal with the effect of non-goal teleologically relevant conditions factored out. Such conditions include forces and boundaries of various kinds, whose effects on the entity's behavior a measurer is not interested in. Further, where teleologically relevant conditions vary, persistence values are calculated over portions of the space where they are roughly homogeneous. In other words, persistence values are local in that they refer to a particular environment-to a particular set of teleologically relevant conditions - in which movement toward and away from the goal is affected in the same way by the same constellation of factors.

Of course, not all environmental factors will be teleologically relevant. For a bacterium swimming in a tube up a chemical gradient, the color of the water in the tube might vary over its length, but if the bacterium's behavior is not affected by the color or the coloring agent- that is, if color is not teleologically relevant-then no environmental partitioning is necessary.

Notice two things. First, the teleologically relevant conditions that require a correction will depend on our interests. For a bacterium that seeks light as well as food, if we are interested in measuring its persistence in its search for food, the presence of light is a potentially confounding factor which we need to correct for when light is present. On the other hand, if we are interested in its persistence in moving toward light, then the presence of food is a potentially confounding factor.

Second, environmental subdivision will sometimes be necessary even in the absence of confounding non-goal factors. Imagine a person who is blindfolded in a large field and trying to 
find a pizza in an open box placed on a table somewhere in the field. Suppose that the person's sense of smell has a threshold such that he cannot smell the pizza at all from distances of greater than 10 meters away, and therefore his persistence beyond that distance is zero. (The maximum range over which the animals can successfully detect or navigate to the goal has been of particular interest in studies such as in honeybee homing behavior [e.g., Pahl et al. 2011].) Now the odor gradient could nevertheless be the same everywhere, and if it is, the environment is technically homogeneous. However, on account of the structure of the person's perceptual capabilities, it is effectively heterogeneous. In such cases, it would be unreasonable to combine moves made at all distances from the goal into the same measure of persistence. Instead, if a threshold is suspected, one could subdivide the field into small subregions, within which all points lie at about the same distance from the goal, and calculate the persistence value specific to each subregion, in this case, to each donut-shaped band.

\section{A Persistence Metric}

We are now in a position to formulate a formal measure. Persistence is:

$$
P=\frac{\frac{G}{N}-R}{1-R}
$$

where $P$ is persistence, $G$ is the number of good moves, $N$ is the total number of moves, and $R$ is the probability given the structure of the space of a randomly moving entity making a good move by chance alone. In other words, $R$ represents the effect of the local, non-goal teleologically relevant conditions, and the equation calculates $P$ with the effect of those conditions factored out. Further, $P$ is understood to be specific to a subdivision of the environment that is assumed or known to be homogeneous with respect to the teleologically relevant conditions. In other words, it is specific to a portion of the environment in which $R$ is assumed or known to be roughly constant. In the one-dimensional case, where the environment is unstructured (no boundaries), half of the available chance moves will be toward the goal, and half will be away, so that $R$ is 0.5 . Notice that in any portion of the space where $R=1$, where the structure of environment only allows movement toward the goal-for example, adjacent to a boundary in a one-dimensional space-persistence is undefined. The two dimensional case is somewhat more complicated. For one thing, $R=1$ and persistence is undefined in corners. As shown in Figure 1, all of the moves available to the particle at location $B$ take it closer to the goal, and therefore for both a randomly moving entity and a goal-directed one, $R=1$, and persistence is undefined. For location $C$, along an edge, $R=0.33$. And for $D$, two of the four possible moves are good ones, so $R=0.5$, as for the unconstrained particle in the one-dimensional case. Location $A$ is an interesting case, in that no boundary constrains it but movement toward the goal can occur along only a single path, along only one of its four possible moves, giving it an $R$ value of 0.25 . In this case, the teleologically relevant conditions include, not just boundaries, but the rules of movement, which require the particles to move along grid lines. In other words, the environment is structured in such a way that the entity is forbidden from moving obliquely toward the goal.

Besides boundaries and rules of movement, there are other ways that teleologically relevant conditions could structure the environment. Various forces might limit the movement of a goal-directed entity, perhaps varying continuously or probabilistically, instead of the all-or-none constraints imposed by boundaries. An example might be a charged particle that moves within an electric field as it pursues some goal. Or an individual driving home while trying to avoid roads with heavy traffic. We do not attempt to calculate $R$ values for such cases, but dealing with them poses no problem in principle. 


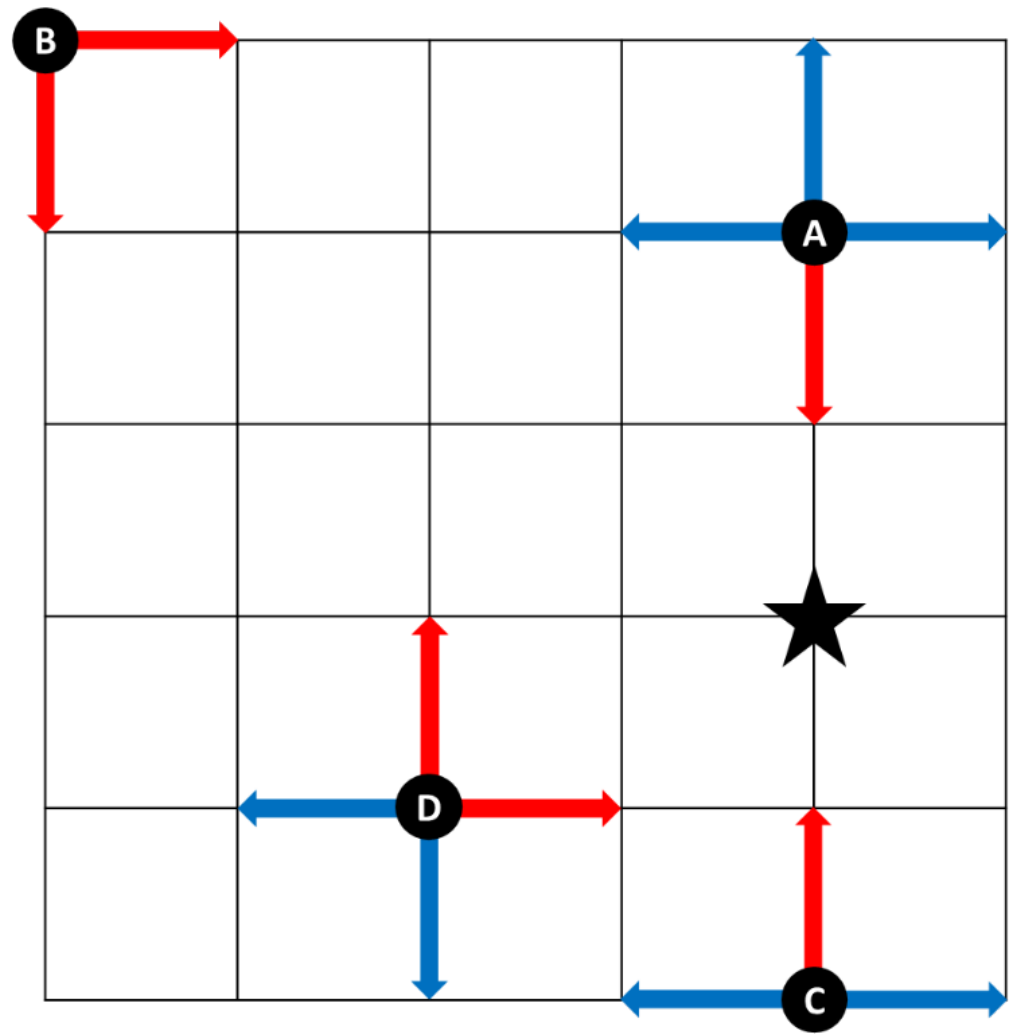

Figure 1: Teleologically relevant conditions, such as edges, affect the way persistence is calculated. In this hypothetical $6 \times 6$ grid, the star represents the goal, and the entities on the four locations- $A, B, C, D-$ can move only along the grid to a neighboring point. The arrows represent all the possible moves that each entity can make on the next turn, the red ones are "good moves" that will reduce the distance between the goal and the entity, and the blue ones are "bad moves" that will increase the distance. Notice that the location of the entity with respect to both the goal and the edge matters. See text for further explanation. 


\section{Plasticity}

In one dimension, in the absence of boundaries and forces, a perfectly goal-directed entity will move directly toward the goal along the shortest trajectory possible. Thus, for such an entity, the length of its trajectory is always equal to the starting distance from the goal, as discussed. In general, for a perfectly goal-directed entity, the plot of the trajectory length versus the starting distance is linear, with an intercept of 0 and a slope of 1 , as long as the starting distance is within the range of directing field. This is maximum plasticity.

For plasticity, the measure we propose is based on the deviation from a slope of 1 . The greater the slope, the less the plasticity. More precisely, plasticity is measured as:

$$
Q=\frac{1}{S}
$$

where $Q$ is plasticity, and $S$ is the slope of the best-fit line in a graph of the relationship-for a given population of entities, or for multiple runs of the same entity-between the length of the trajectory the entity follows to get to the goal and its initial distance from the goal (Figure 2).

Figure 2 shows how plasticity is calculated for goal-directed entities. The data points represented by solid circles correspond to entity 1 and those with open circles to entity 2 . Each data point represents the median trajectory length measured from a set of trajectories that have about the same starting distance. Then, a linear regression is computed for each of the two sets of medians, and plasticity is calculated by dividing 1 by the resultant slope (in the figure, $a$ and $b$ ). In this case, the plasticities $(Q)$ for entities 1 and 2 are $1 / a$ and $1 / b$, respectively. Entity 1 has a higher plasticity than entity 2 , meaning that, on average, a given increase in starting distance of entity 2 resulted in a greater increase in trajectory length than did the same increase in starting distance for entity 1.

Plasticity measures how close a system's trend of trajectory length versus starting distance is to the trend expected from perfect plasticity, in which trajectory length is always equal to starting distance. The less, on average, a given increase in starting distance increases trajectory length, the more plastic this system is.

Ideally, a measure of plasticity would be sensitive to the structure of the space, as our persistence measure is, and while such a correction is conceptually straightforward, it is beyond the scope of this paper.

\section{Demonstration 1: Goal Directedness in a Bacterium}

The metrics were applied to measure persistence and plasticity in a bacterium, E. coli, as it moved along a linear gradient of chemoattractant ( $\alpha$-D,L-methylaspartic acid, MeAsp). (Data were provided by Dr. Remy Colin and Dr. Victor Sourjik at the Max Planck Institute for Terrestrial Microbiology, see acknowledgments.)

\section{Procedures}

The experimental setup is a microfluidics device consisting of an open-ended channel attached on one side to a reservoir with a high concentration of chemoattractant and on the other side to one with zero concentration, creating a linear concentration gradient along the channel (see Micali et al. 2017 for further details). The bacteria follow a so-called run and tumble trajectory, in which they move in a straight line for some distance (a run) and then re-orient randomly (a tumble) every so often. Straight runs are short when the chemoattractant concentration 


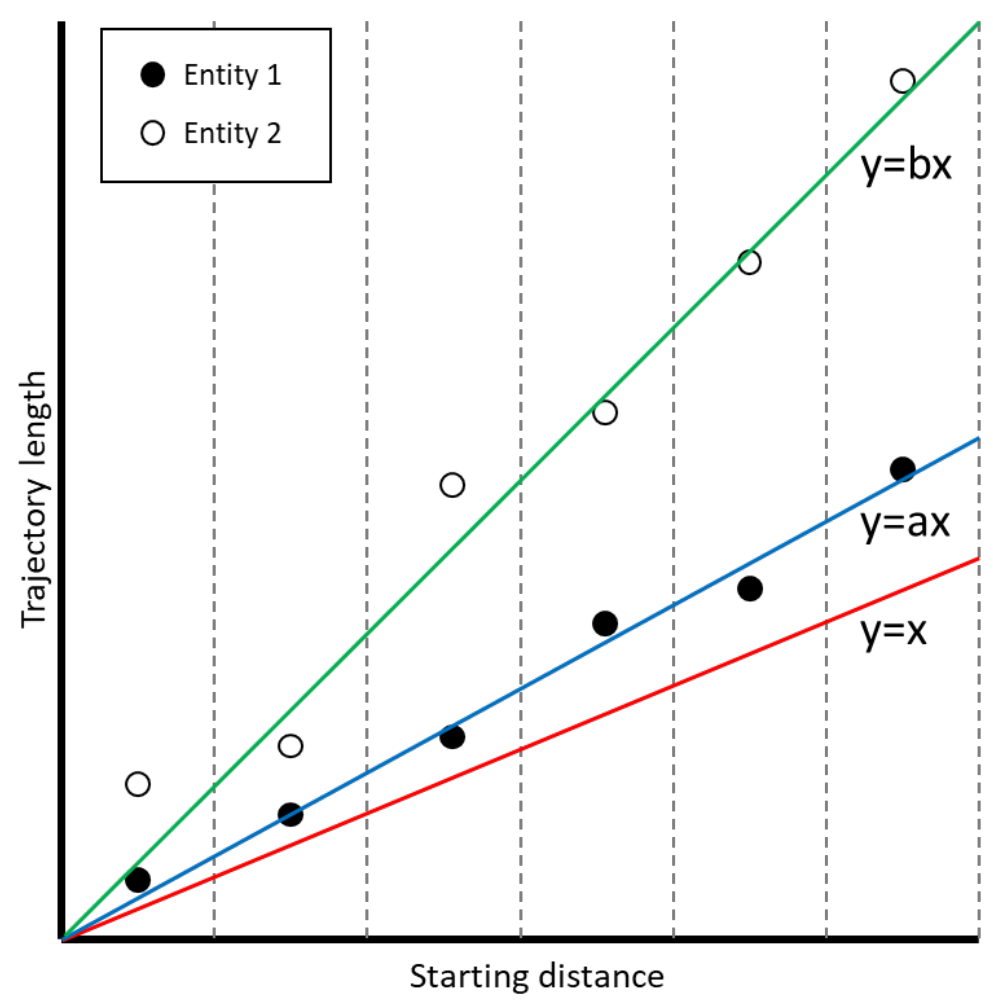

Figure 2: A hypothetical graph of trajectory length versus starting distance for calculating plasticity. See text for further explanation. Entity 1's slope is less than Entity 2's, and closer to $1.0(y=x)$, and therefore Entity 1 is more plastic.

is low, longer when the concentration increases. The trajectories of the bacteria are captured on camera, and their positions in a linear coordinate system are digitized (Micali et al. 2017). Figure 3 shows the setup schematically. In this coordinate system, the highest concentration occurs at $x=0$ and lowest at $x=1022$, so the bacteria move-running and tumbling-from right to left. The physical setup is actually three dimensional, in that the bacteria are free to move in any direction, at least within the channel. But for present purposes we treat it as one dimensional, taking into account only the $x$ component of their movements, only movement along the concentration gradient. A good move- a move toward the goal-is considered to be one that takes a bacterium to a smaller $x$ value. Notice that there are no boundaries in the $x$ direction, and therefore for present purposes their movements are unconstrained.

\section{Persistence}

Before measuring persistence, the data were filtered, removing all trajectories that lasted less than 2 seconds. $X$ coordinates of each of these longer trajectories were recorded every 0.5 seconds and used to determine whether each 0.5 -second-move was good or bad, toward the goal (the direction of smaller $x$ coordinate) or away from it. The concentration of chemoattractant changes along the $x$ axis, so the $x$ axis was divided into 10 bands, each of width 100, plus 1 band with width of 22. The assumption is that variation in concentration of chemoattractant is relatively low within each band. Thus each band is effectively environmentally homogeneous and a persistence value can be calculated for it.

The statistics of good and bad moves obtained from each band were entered into equation 1 . No physical structure or force constrained the E. colis direction of movement in the $x$ direction, 


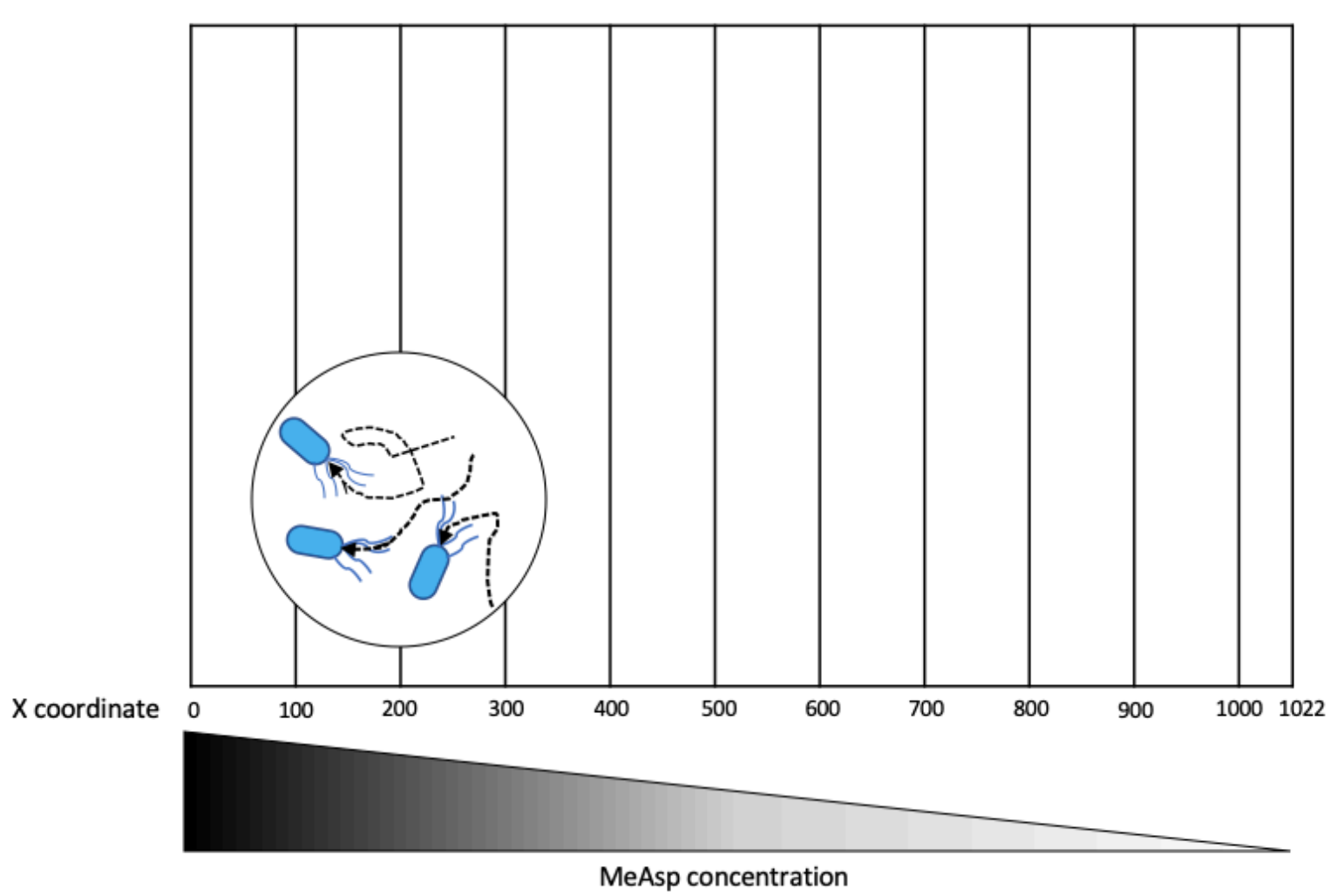

Figure 3: The experimental setting for E. coli chemotaxis developed by Micali et al. (2017). E. coli are released into the microfluidics device-essentially a narrow channel-with a fixed, linear gradient of MeAsp, a non-metabolizable chemoattractant. The movement of $E$. coli is digitized on a coordinate system, in which the lowest concentration of MeAsp is on the right, at $x=1022$, and it increases along the $x$ axis, reaching the highest value at $x=0$. (The small circle in the bottom left is a cartoon showing magnified, hypothetical trajectories of several E. coli.) The camera captured only the area within the channel of the microfluidics device, so contrary to the figure, E. coli's movements are not actually constrained by the apparent boundaries in the coordinate system. After the gradient becomes stable, bacterial motion data are collected 3 times with 1-hour interval for each of 3 repeats (see Micali et al. [2017] for further detail). 


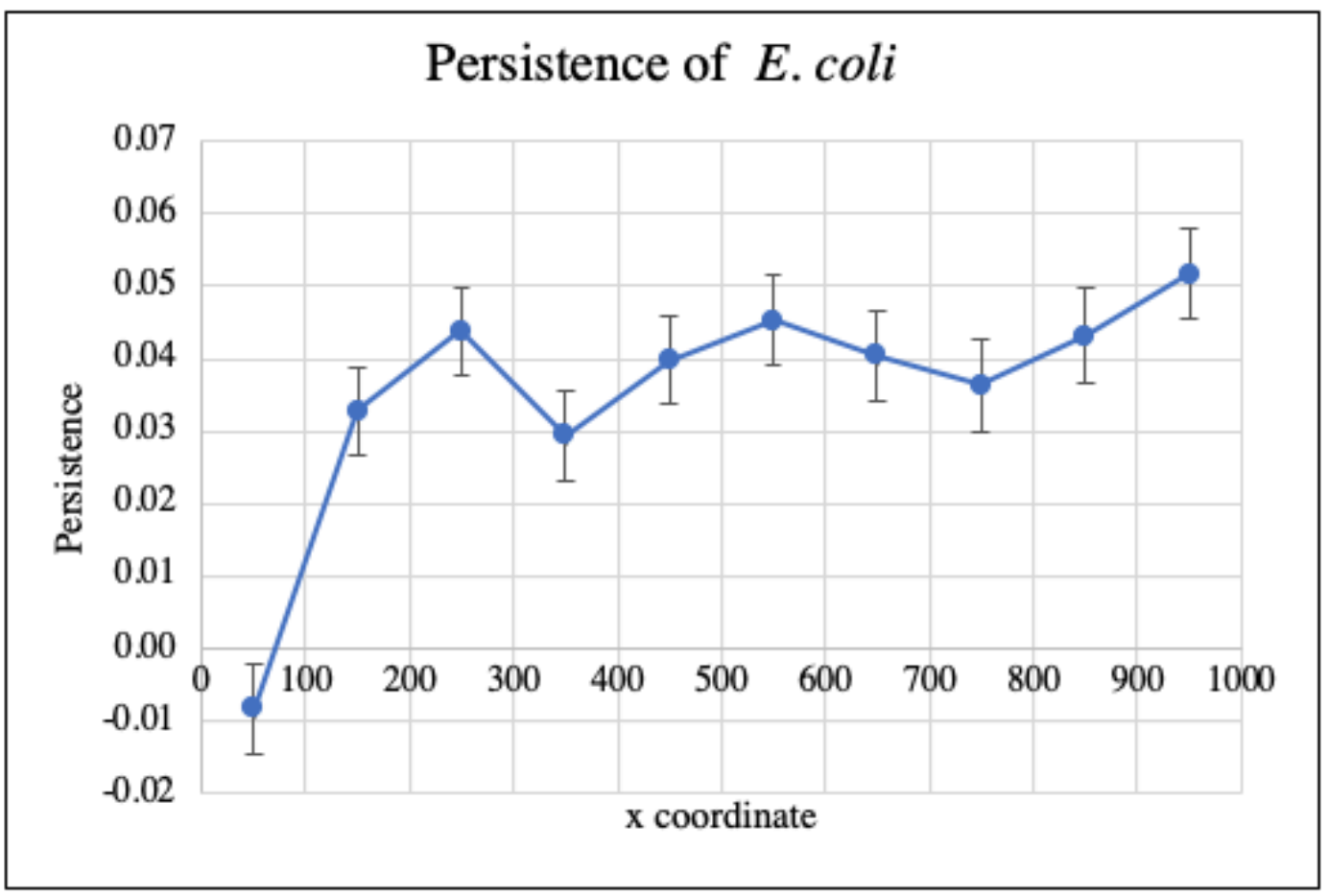

Figure 4: Persistence of E. coli along a linear MeAsp gradient. Each persistence value represents the local bias of the unit-moves that occurred within a specific band along the MeAsp gradient. The persistence value of about 0 that was observed at the band of $0-100$ indicates a ratio of good moves to total moves that is expected by chance alone. In the rest of the space, E. coli demonstrated consistently low persistence, around 0.04, except for the last band (1000-1022) with the lowest MeAsp concentration that shows significantly higher persistence (not shown in the current figure; see Appendix). Lower and higher persistence values observed at each end are probably due to a technical bias in sampling, since the trajectories toward the higher concentration near the edge at $x=0$ and those toward the lower concentration near the edge at $x=1022$ are more likely to be interrupted and lost in the filtering process. Error bars represent one standard error.

and therefore $R$, the expected probability for a randomly moving entity to make good move by chance alone, was assumed to be 0.5 in all bands.

Persistence values ranged from 0 to 0.15 . (A negative calculated persistence value can arise only by chance and is assumed to correspond to zero persistence.) Most of the bands between $x$ coordinate of 100 and 1000 demonstrated similar persistence measures around 0.04 (Figure 4).

\section{Plasticity}

To measure plasticity, an imaginary line at the $x$ coordinate of 300 was arbitrarily designated as the goal line. Only the trajectories that started at lower concentration level (starting $x$ coordinate greater than 300), lasted at least 2 seconds, and reached the goal line were used to calculate plasticity. For the trajectories that satisfied these conditions, the starting distance from the goal line and the trajectory length were calculated. The trajectories were divided into groups based on their starting distances, so that each group included the trajectories with starting distances over a range of 10 units. For example, one group consisted of those bacteria that started between 300 and 310, a second group was those that started between 310 and 320, and so on. Then, the 
median trajectory length was obtained for each group to generate the scatter plot and the linear regression in Figure 5. The analysis for plasticity was limited to those starting between 300 and 400. The slope of the linear regression generated by the median trajectory lengths from different starting distances was $1.401 \pm 0.136$. Applying equation $2(Q=1 /$ slope $)$ gave a plasticity value of 0.714 for the bacteria that moved toward the goal line at 300 .

The calculation was repeated using a goal line at $x=100$. The slope of the linear regression generated by the median trajectory lengths from different starting distances was $1.994 \pm 0.140$ (Figure 5). Thus, again applying equation 2, the plasticity value for the entities that moved toward the goal line at $x=100$ was 0.502 , less than for trajectories that moved toward $x=300$.

\section{Demonstration 2: Human Goal Directedness}

A $17 \times 9$ grid with a coordinate system was drawn on a rectangular metal plate, with $(0,0)$ at the center (Figure 6). Below the metal plate, a source of heat was randomly placed so that a temperature gradient was formed on the metal plate. After sufficient time had passed for the gradient to reach equilibrium and remain stable, a human subject, unaware of the location of the heat source, was asked to locate the heat source using only the sense of touch, a single finger placed on the metal plate (although vision played a role in guiding movement of the finger from one grid location to the next). The subject always started from $(0,0)$ and moved only along the grid lines, until the subject reached the heat source. The trajectory was recorded as a series of coordinates. A run of the experiment ended when the subject reached the heat source, and a new run was begun with the heat source under a new location, hidden from the subject. After the heat gradient from the previous run had dissipated and a new one was established, the subject was again asked to locate the heat source. The experiment was repeated 40 times. For each run, the location at which the subject started to sense the presence of heat gradient was recorded.

Notice that this demonstration and the first are different in that the first involved multiple independent individuals while this involves a single individual doing multiple runs. They are similar, however, in that the experiment setup is a constant - among individuals or among runsand in both cases persistence and plasticity are calculated as averages over multiple results.

\section{Procedures}

For measuring persistence, Euclidean distances between the goal and the subject's consecutive locations on the grid were compared to determine whether a move was good or bad. If the distance between the goal and the subject decreased after a move, that move was considered good. Then the range of distances was subdivided into 16 "bands," each 1-unit distance wide (approximately $2.5 \mathrm{~cm}$ ). For instance, moves that started between 1-unit and 2-units distance away from the goal were combined to calculate a single persistence value. Likewise, each of the other bands included the moves started between 2 and 3, 3 and 4, and so on. In this setup, the structure of the environment, i.e., the grid and its edge, significantly constrained movement, so $R$ values were calculated, in this case as an average for each distance band. Thus if there were two moves that occurred at distance 1-to-2 away from the goal, and the individual probabilities of making a good move by chance alone were, say, 0.5 and 0.67 , then $R$ for the set of moves that occurred at 1-2 distance away from the goal was calculated as 0.585 . This tactic amounts to a kind of coarse graining of the constraints in the system.

For calculating plasticity, the starting distance was calculated as the Euclidean distance between the goal and the starting point, and trajectory length was measured as the number of steps taken to reach the goal. 


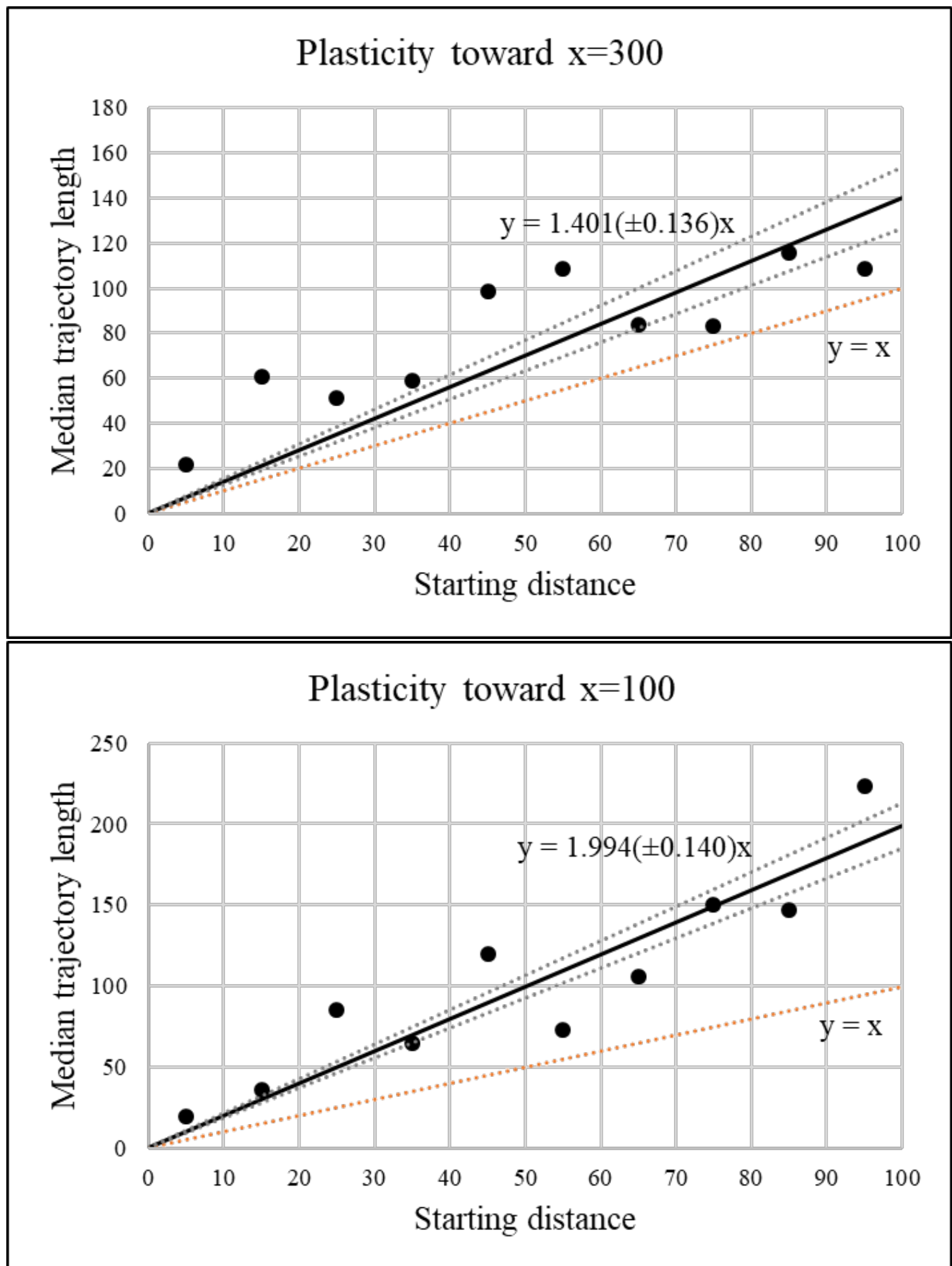

Figure 5: Plasticity of E. coli moving along a MeAsp gradient. Plasticity measures were obtained for two different designated goal-lines, at $x=300$ and $x=100$. All trajectories that reached the goal-lines were grouped based on their initial starting distance from the goal line, so that those starting at a distance between 0 and 10 were grouped together, 10 and 20, 20 and 30, and so on. The points on the graph represents the median trajectory length of each group, and the linear regressions for the median values are shown as a solid line. Plasticity is then calculated by dividing 1 (the slope of the $y=x$ line) by the regression slope (equation 2). When the goal-line is defined at $x=300$, plasticity was calculated to be 0.714 . When the goal-line is defined at $x=100$, it was 0.502 . Gray dotted lines represent a standard error of the slope of the linear regression.

○ OPEN ACCESS - PTPBIO.ORG 


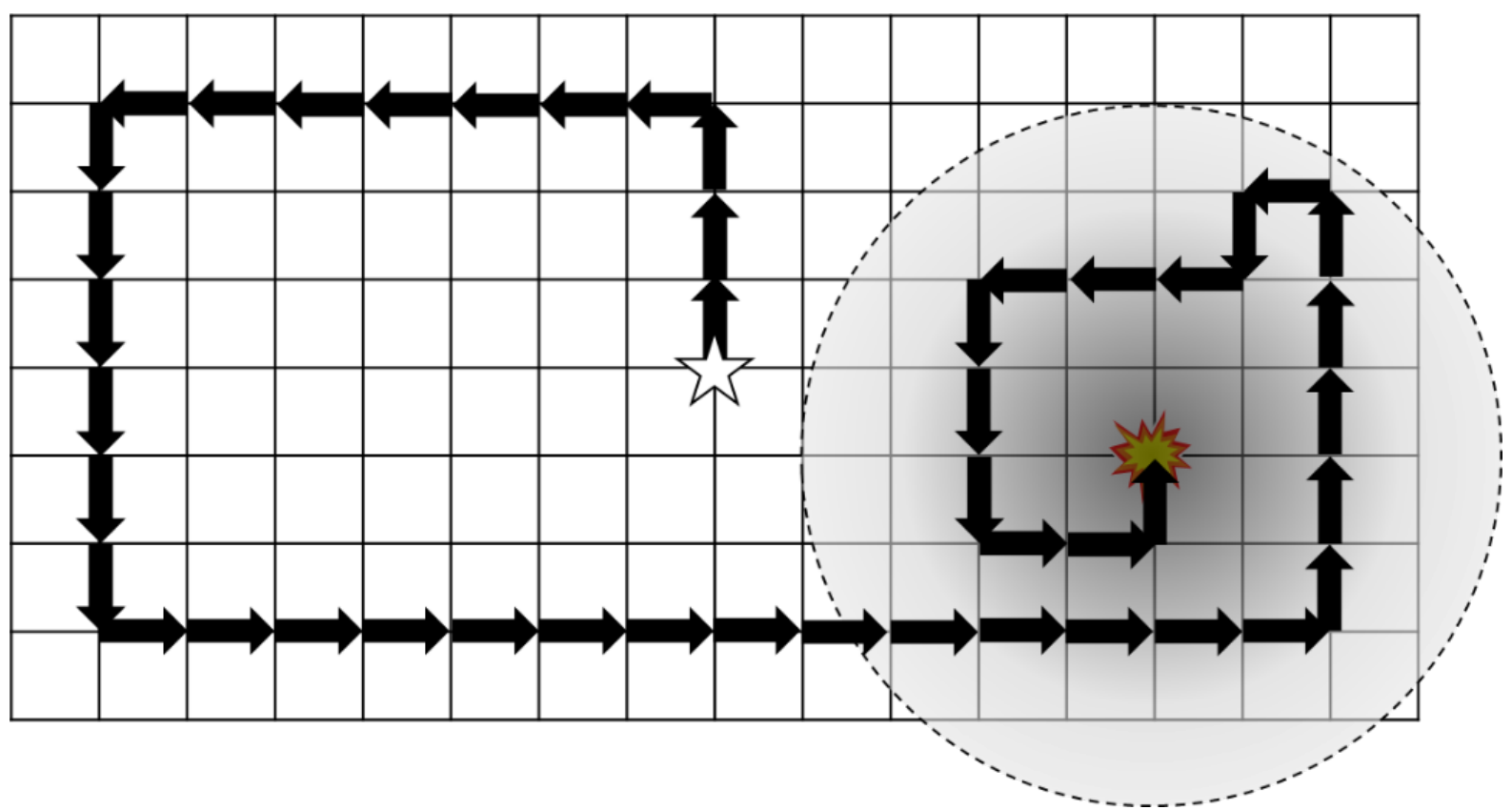

Figure 6: The experimental setup for assessing human goal directed movement. The star represents the center of the 17 by 9 inch coordinate system and the starting point for all runs of the experiment. The distance between neighboring points is defined as 1 unit-distance (about $2.5 \mathrm{~cm}$ ). The yellow icon represents a possible location of the heat source for a given run. The shaded circle represents the average range of the heat gradient where the subject was able to sense the presence of the heat gradient.

\section{Persistence}

Overall, persistence values at moderate distances (up to 11-12 units) ranged from 0.08 to 0.47 (Figure 7). For 40 repeats, the average distance at which the subject reported sensing the heat gradient was 3.92 (Figure 6), and in fact the moves that occurred within that radius had significantly higher persistence values (Figure 8). Outside that radius, the subject moved randomly or, in some cases, moved in a way calculated to cover unexplored territory, producing betterthan-random persistence. All of the persistence measures in this setting are probably boosted by this effect, an effect that is not accounted for in the calculation of $R$. Some proportion of the moves at all distances occurred near the edge. Even if the subject was only one or two steps away from the edge, was not physically constrained by the edge, and therefore had the choice to move toward the edge, he was more likely to decide to move away from it, in a way calculated to cover more unexplored territory, leading to higher persistence. In effect, there is a psychological process that leads to an underestimate of $R$. There could also be a "corner bias," arising from the fact when the goal is placed in a corner, there are fewer approach routes available. In principle, these edge biases could be quantified and incorporated into the metric as boundary effects, but we do not attempt that here.

Negative persistence was measured in the ranges between the distance of 12 and 13, and between 13 and 14. As discussed, negative persistence is never the expectation, when the goal is attractive (as opposed to repulsive), and the negative values here are likely due to the small sample size, 8 and 10 respectively (see Appendix). The extremely high persistence values calculated at distances greater than 14 are likely due to the combination of small sample size and edge effects, discussed above, that are not accounted in the calculation of $R$. 


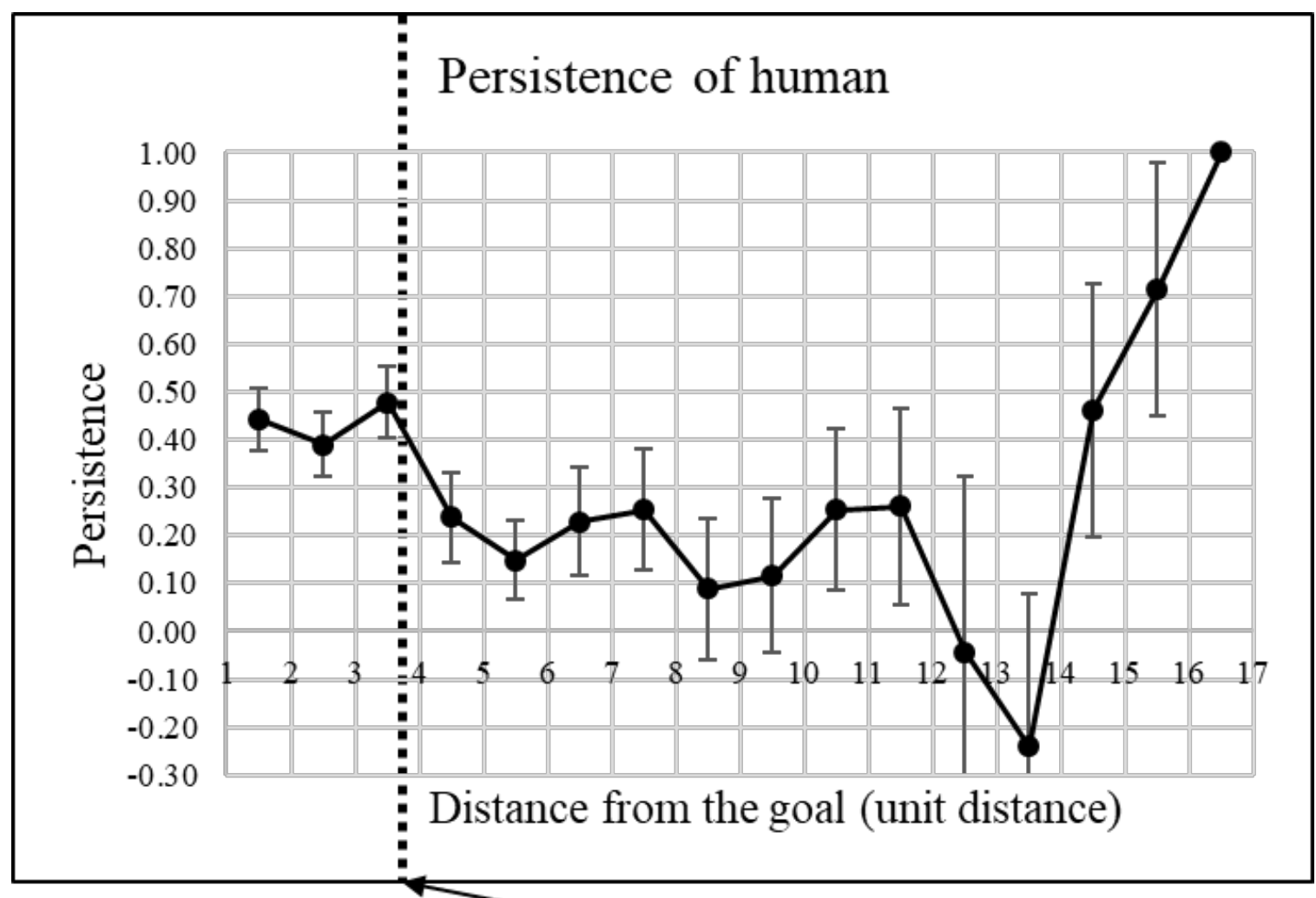

\section{$\mathrm{X}=3.9215$ : Average distance at which the subject started to sense the presence of heat gradient}

Figure 7: Persistence of a human searching for a heat source under the grid system. Each persistence value represents the local bias of the set of moves that occurred within a certain range of distances from the goal. Overall, persistence values in this experimental setting were quite variable. Assuming the average radius of the detectable part of the heat field that formed on the metal plate was 3.922 unit-distance, the moves that occurred within that range were significantly more persistent than those that occurred out of it (see also Figure 8). The moves that occurred at large distances away from the goal (14-17) demonstrated seemingly high persistence, but it is likely due to the combined effect of small sample size and the unaccounted-for edge effect (see text). 


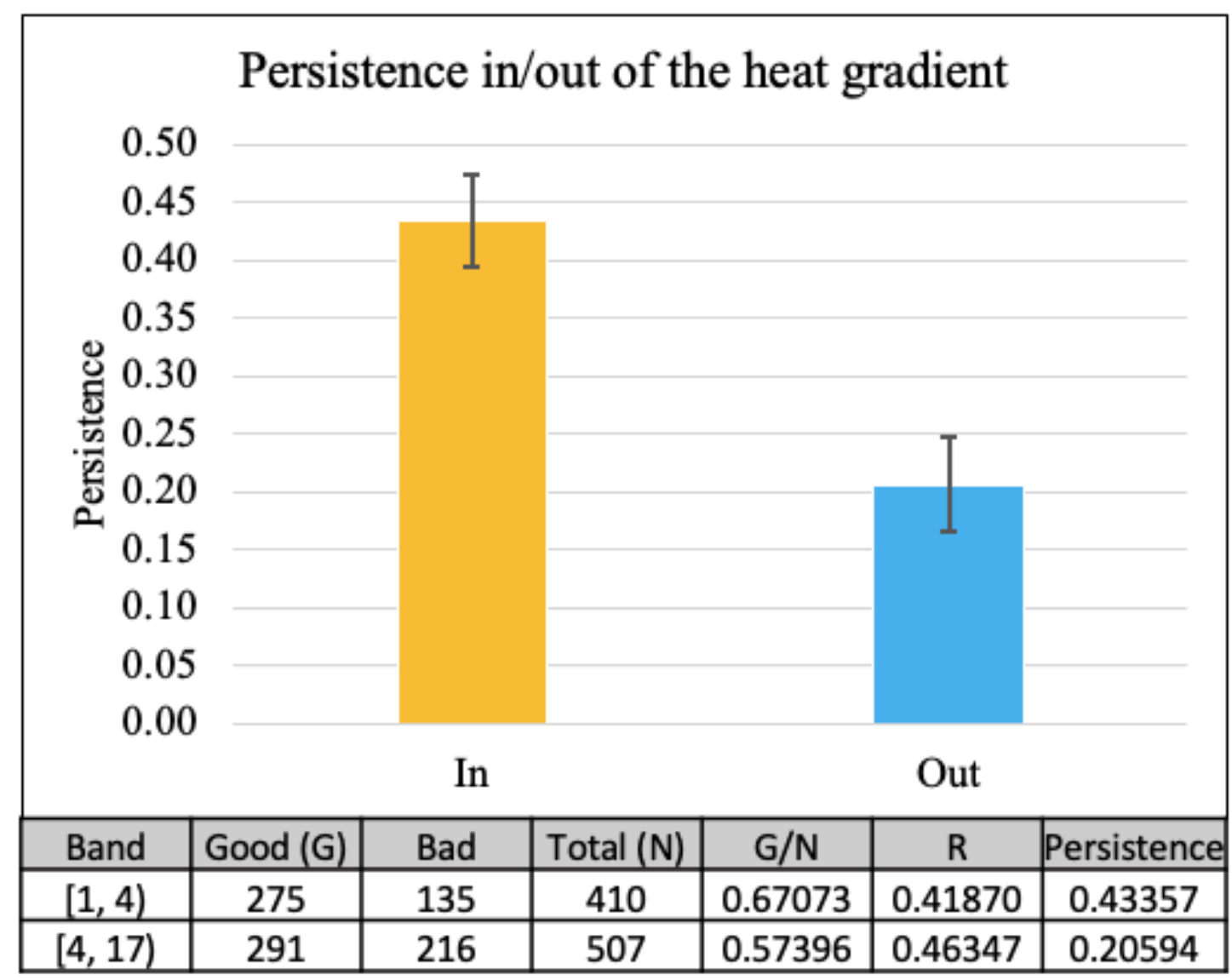

Figure 8: The moves that occurred within the detectable range of the heat field were significantly more persistent than those that occurred out of it. Within the detectable range, the field directs the trajectory whereas the moves outside this range will be either random or systematic. By systematic we mean that the presence of an edge, perceived visually, systematically increases persistence, because the subject is less likely to move toward the boundary even when given the choice to do so. This probably accounts for the fact that persistence values averaged significantly greater than zero outside the detectable range. 


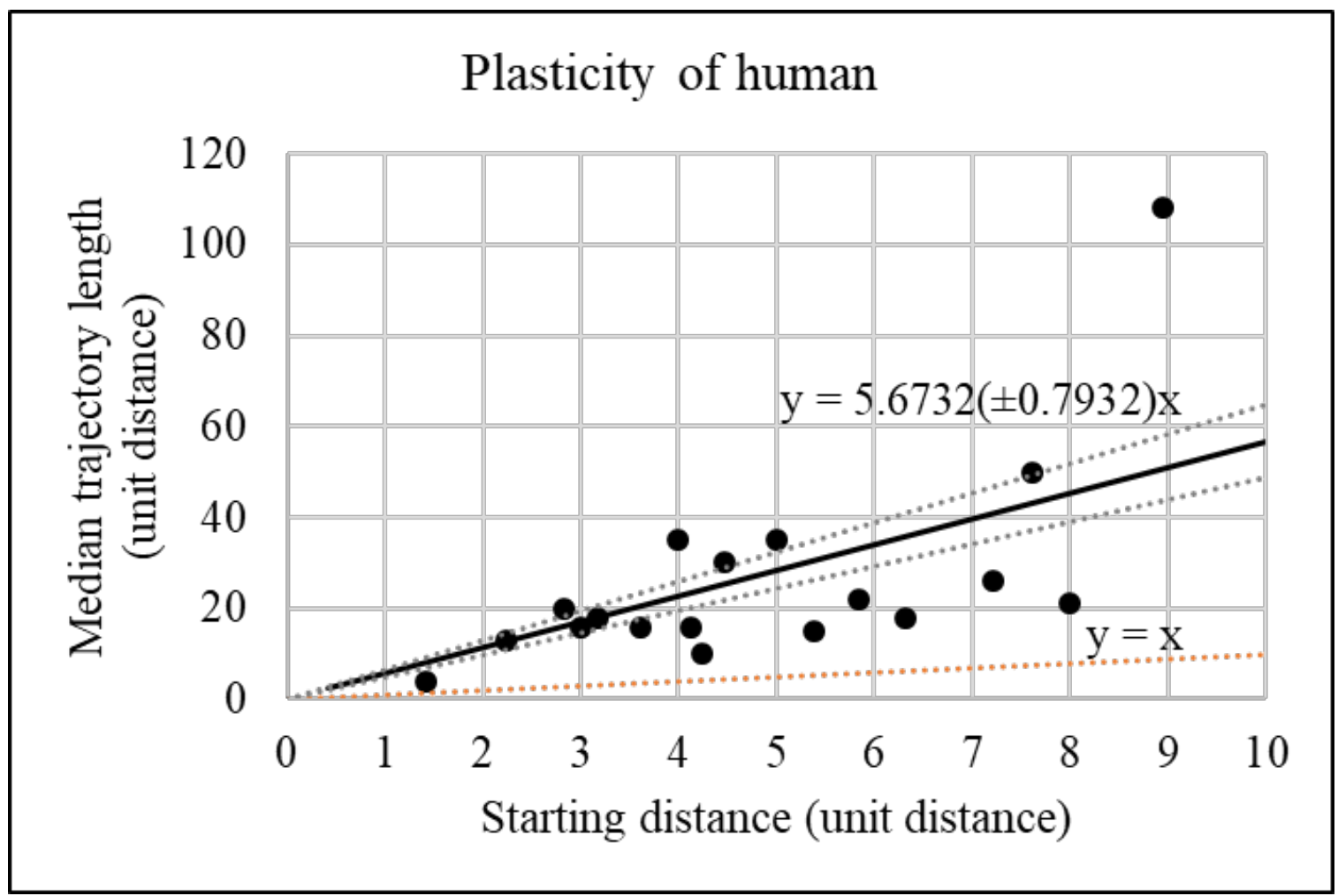

Figure 9: Plasticity of human using the sense of touch to search for a heat source in a grid system. Since the starting distances of the trajectories were discretely distributed, all the trajectories that had the same starting distance were grouped together. Then, medians from these groups were obtained to generate a linear regression with the slope of 5.673. Thus, the plasticity was calculated by dividing 1 (the slope of the $y=x$ line) by the regression slope to yield a plasticity value of 0.176 (equation 2). Note that this value is much smaller than the plasticity values of $E$. coli in Demonstration 1 (Figure 5). Gray dotted lines represent a standard error of slope for linear regression.

\section{Plasticity}

For each of 40 trajectories, the Euclidean distances between the goal and the starting point (0, 0 ), i.e., starting distance, were calculated. The trajectory length was calculated as the number of steps each trajectory took to reach the goal. Unlike the $E$. coli demonstration, all starting distances were discretely distributed due to the grid setting. Thus, the medians were obtained from the groups of trajectories which had a common starting distance, to generate the scatter plot and the linear regression (Figure 9). The slope of the linear regression generated by the median trajectory lengths from different starting distances was 5.673 \pm 0.793 . As a result, the plasticity value for the human moving toward the source of heat was $0.176(Q=1 /$ slope, equation 2) $)$.

\section{Caveats and Limitations}

It is obvious, but worth mentioning anyway, that the measures of persistence and plasticity are context specific. The measured values of persistence and plasticity for the bacterium refer only to its pursuit of a particular chemoattractant in a microfluidics device, and those for the human refer to a subject seeking of a heat source under a metal plate using his sense of touch. Different experimental settings and motivators require different adjustment strategies for measurement and are expected to produce different values. 
Beyond this, both demonstrations have uncertainties arising from the limitations of the experimental design. We have already mentioned the unmeasured bias away from the edges in the human demonstration. In the $E$. coli demonstration, since it was unlikely that every single $E$. coli would reach the designated goal line, only the small subset of trajectories that succeeded in reaching the goal line within the given experimental time frame were used for analysis. Therefore, $E$. coli with smaller starting distances and smaller trajectory lengths were more likely to be included in the data, while ones with greater starting distance and trajectories might have not been properly represented. On the other hand, medians are fairly robust to outliers, and there is no reason to think that non-included trajectories would produce any systematic bias in the estimate of the slope, and so differences arising from inevitably different details in the measurement procedure will probably be insignificant.

More generally, the metrics themselves have certain limitations. First, they are applicable to limited range of goal-directed behaviors. For instance, they cannot be applied to goal-directed behaviors that involve repulsion such as a rabbit running away from a fox. Also, it would be difficult to use them to measure persistence and plasticity in entities that have internal goals (e.g., human desires) and unobservable behaviors (thoughts motivated by desires). And since the introduced metrics take a statistical approach, a sufficient sample size and some degree of variability within samples are necessary. For instance, if all the trajectories have the same starting distance, then it will be impossible to measure plasticity. Further, application of the metrics is limited to entities in which the governing mechanism is stable both in its structure and in its response profile, and in which the mechanisms are similar from one entity to the next. Thus, the metrics cannot be used when entities are highly variable in their sensitivity to the environment. For instance, we assumed that all of the E. coli in Demonstration 1 had about the same response profile.

Another limitation is that measurement requires a fair amount of knowledge about the environment. For one thing, one has to know the location of the goal. One also has to know the nature and distribution of the teleologically relevant conditions. For instance, to calculate $R$, the locations of boundaries must be known. In the demonstrations, this was not an issue because entities were either unconstrained, as in E. coli, or constrained in a way that enables easy mathematical estimation of $R$, as in the human demonstration. Also, in the $E$. coli demonstration, interactions among entities could play an important role in goal directedness, but as formulated, the metrics cannot take such interactions into account. The assumption is that every trajectory is independent of every other.

Lastly, one major assumption that the metrics require is that the relationship between the entity and the field is stable. If some environmental factor changes dramatically during a trajectory, or if some property of the goal-directed entity changes, the result will not be meaningful.

These limitations suggest various ways in which the metrics could be modified or extended, to accommodate repulsion, or to accommodate complicated situations with multiple goals. Furthermore, as presently configured the metrics require binary data, taking into account only whether a move is toward the goal or not, whether it is good or bad. It ought to be possible to design similar metrics in which moves are weighted, metrics which take into account the angle of a move with respect to the goal, its degree of goodness or badness, so to speak.

\section{Discussion}

This paper attempts to develop a unified approach to the study of goal directedness by introducing metrics that are applicable to any entity that shows the signature features, persistence and plasticity. In principle, these metrics are universal in that they can be applied to any behavioral

๑ OPEN ACCESS - PTPBIO.ORG 
trajectory in any biological system. Persistence and plasticity can be measured in any data set that records goal-directed trajectories, from salmon swimming upstream, to birds migrating, to snails crawling up a beach. Here we applied the metrics to movements of organisms in space. But persistence and plasticity can also be measured in data on physiological changes occurring over time within an organism, such as changes in metabolic rate, hormone concentration, or neural activity. In these, movement occurs in a state space defined by the parameters of the system. Persistence is bias in the direction of change toward a target state of the system, and plasticity is the directness or efficiency with which the system's state moves toward the target state. The metrics could even in principle be applied at higher levels of organization to assess the goal directedness of mental activity, the "movement" of thought, so to speak, on a trajectory that spans a number of mental states of some kind. (An "idea space?") It is not obvious how to define such a space, but the point is that if it could be defined, and if data could be gathered (perhaps by direct reporting by human subjects), the metrics could be used to assess persistence and plasticity for various purposeful activities, under various alternative conditions. In sum, the metrics assess these two properties of goal directed entities using their behavioral trajectories and can be applied to any population of trajectories for which trend data can be gathered.

Armed with these metrics, comparative studies become possible. One could, for example, use them to compare the goal directedness of two related species of migrating bird. Does the one with larger brain size show greater persistence or plasticity? And comparisons could in some cases be done across a wider taxonomic gap. In an experimental setup with food as the goal, is a hungry dog more persistent or less, more plastic or less, than a hungry person? Or in a non-biological context, one could compare the goal-directedness of successive generations of robots guided by artificial intelligence. Is the later generation of floor-sweeping robot more persistent or more plastic than the earlier?

The metrics can also be used to investigate the causes and correlates of goal-directed behavior. How does changing the concentration gradient, or changing the chemo-attractive substance, affect the persistence and plasticity of $E$. coli? Are certain kinds of human behaviors less persistent, or less plastic, when we are tired?

Finally, the metrics could be used to investigate evolutionary trends. Does persistence tend to increase over time in evolving lineages? Does plasticity tend to increase in, say, highly variable environments? Under what circumstances might goal directedness be disfavored in evolution? Are there circumstances under which selection acts against persistence and plasticity, perhaps on account of high computational costs?

Of course, empirical studies are subject to the same limitations as all comparative studies in biology. Goal directedness is like fitness in that it is simultaneously an organism-dependent and environment-dependent property, and comparisons between entities in which both differ dramatically are not meaningful. For this reason, we do not make much of the difference in persistence and plasticity values revealed here between the bacterium and the human. The bacterium had much lower persistence than the human, with values mostly lower than or near 0.05 while the human values ranged from 0.08 to 0.47 . For plasticity, both values calculated for the bacterium (0.714 and 0.502) were high compared to the value obtained for the human, 0.181 . But of course, these values were computed in very different experimental setups, under very different sets of teleologically relevant conditions. A meaningful comparison between the species would require that the experiments be performed in setups that were similar, at some level of abstraction, more precisely in setups that are similar in ways that are teleologically relevant to the species involved. The goal-directed capacities of a human immersed in a MeAsp gradient are not engaged to the same extent, or in the same way, as a bacterium's in that environment. (For the human, the issue would be drowning, rather than food.) Thus designing an experimen- 
tal setup that allows a meaningful comparison between two species that are quite different from each other would be a real challenge. In any case, the point here was not to do a comparison between bacteria and humans, but to demonstrate the metrics, to show the huge breadth of their domain of application.

\section{Discreteness and Unity}

The results obtained are useful for making two conceptual points. First, goal directedness can be understood as a continuous variable. In the modest literature in the philosophy of biology on goal directedness, it has been implicitly treated as discrete, just as function is treated, as either present or not. Now it is theoretically possible that goal directedness could have been manifest in biological systems as a discrete phenomenon. But everyday experience suggests the opposite. In most simple tropisms, such as sunflowers tracking the sun across the sky and moths moving toward light, the behavioral responses to goals seem to be graded. The same goes for more complex goal-directed behaviors, such as a rabbit finding its way into a fenced garden or a person driving home after a long day's work. Degree of goal directedness varies continuously with time and circumstance. The evidence is impressionistic, of course, but it speaks loudly. And the results of the demonstrations above support these impressions. In particular, persistence and plasticity vary continuously in their intensity from one context to another. Persistence in the human was not simply 0 or 1 . It varied with distance from the heat source. The plasticity of the bacterium varied with the chemoattractant concentration.

Second, goal directedness has been implicitly treated as unitary, as a single phenomenon, rather than an alloy or composite of multiple different phenomena. But the results here suggest that it has at least two components, persistence and plasticity, and that they are to some degree independent of each other. Persistence can be low while plasticity high, as in the bacterium demonstration, and persistence can be high and plasticity low, as in the human demonstration. In other words, it looks like persistence and plasticity are capturing different things, different aspects of goal directedness. If that is right, then we need to think about goal directedness, not as a unitary concept but as a composite one. Just as there are multiple types of intelligence (Gardner 1983), there might be multiple types of goal directedness, each to some degree independent of the others, and each representing a qualitatively different capacity.

Importantly, no claim is made here that persistence and plasticity are the only components of goal directedness, the only signature behaviors. In principle, there could be many more. For example, there could be a component corresponding to something like "stick-to-it-iveness," the consistency of an entity's persistence over time. Plasticity too could vary as a function of time, as well as distance. Many more can be imagined, some doubtless worth investigating, and metrics for these would need to be devised.

We make one final point. Nothing in the present study rules out the possibility that necessary and sufficient conditions for goal directedness will eventually be found. In the terms we use here, a necessary and sufficient condition for goal directedness might be manifest as a threshold on the persistence scale. Or some critical combination of persistence and plasticity could be discovered to be necessary and sufficient. In any case, our purpose here is not to take a position on this issue but to put the search for necessary and sufficient conditions on hold. It is to take the study of goal directedness in a new direction, to show that goal-directed systems can be studied empirically and in a unified way. 


\section{Appendix}

\section{Step by Step Procedure for Measuring Persistence}

The equation for persistence is (equation 11):

$$
P=\frac{\frac{G}{N}-R}{1-R}
$$

Here, $P$ is persistence, $G$ is the number of good moves, $N$ is the total number of moves, and $R$ is the expected probability for a randomly moving entity to make a good move by chance alone, given the structure of the space. All parameters are understood to be specific to a homogeneous subdivision of the environment, representing the group of moves that occurred within it. Likewise, both $N$ and $G$ refer to the moves that occurred within that area. The measurement process is as follows:

1. Designate a subdivision of the environment for which the measurer intends to calculate a persistence value.

2. Count the total number of moves that occurred in that subdivision, including moves both toward and away from the goal ("total" moves, $N$ ).

3. Count the number of moves that occurred in that subdivision that reduced the distance to the goal ("good" moves, $G$ ).

4. Divide the number of good moves by the number of total moves in the area of interest $(G / N)$.

5. Calculate $R$ and, then, $P$ for that subdivision.

As discussed, persistence, as well as $R$, is a local value, which may vary across the space. However, in some cases, it is possible to characterize persistence over a larger heterogenous space. For example, in Figure 1, an $R$ for the whole space can be calculated as a weighted average. In this case, if the same number of moves occurred only at the four locations with letters $(A, B, C, D), R$ for the entire grid environment will be average of all four individual $R$ values, $(0.25+1+0.33+0.5) / 4=0.52$. In effect, we are averaging across a range of different teleologically relevant conditions.

Consider a somewhat more complex example, one that is similar to the demonstrations. Suppose that the environmental conditions that affect goal directedness vary, not just as a function of the proximity of boundary, but also as a function of distance from the goal, as occurs in gradients. We could then calculate $P$ values for the specific subdivisions of the environment, each corresponding to the set of points with a common distance from the goal. Then there would be three distinct $P$ values: one for the move that occurred at $B$, another for the move that occurred at $D$, and a third for the moves that occurred at $A$ and $C$, which are considered together because they lie at the same distance from the goal, two steps away. For the calculation of the third $P$ value, the appropriate $R$ value will be the average $R$ value of all the moves that occurred at a two-unit distance away from the goal, i.e., $R$ will be $(0.25+0.33) / 2=0.29$. Now imagine that, over time, 4 additional moves occurred only at $C$, whereas there were no additional moves in other locations. The measurer again wants to calculate the specific $P$ values for the subdivisions of the environment. This time, for the calculation of $P$ value for the moves that occurred at a two-unit distance away from the goal, $R$ will be $(0.25 * 1+0.33 * 5) / 6=0.317$. 


\section{Step by Step Procedure for Measuring Plasticity}

The equation for plasticity is: $Q=1 /$ slope (Equation 2 ). For each entity, measure the starting distance and the length of the trajectory the entity follows to the goal.

1. Subdivide the set of trajectories based on their starting distances, so that the trajectories within a certain range of starting distances are grouped together.

2. Obtain a median trajectory length for each group, and use those medians to generate the scatter plot, whose $x$ axis is the starting distance and $y$ axis is median trajectory length.

3. Generate a linear regression with $y$ intercept of 0 and calculate plasticity as $Q=1 /$ slope.

Although this paper just introduces one possible way to plot trajectory lengths versus starting distances and to measure deviation from perfect plasticity, alternative methods are possible. The above procedure takes advantage of the fact that medians are relatively less affected than means by the uneven, biased distribution of starting distances and outliers that may complicate the process of calculating plasticity. Moreover, distributions of trajectory lengths at any given starting distance are likely to be skewed because there is a limit to how short the trajectory can be-trajectory length cannot be shorter than starting distance-but no limit to how long it can be. This skewed distribution of trajectory lengths at a given starting distance make the mean unstable as a measure of central tendency and dependent on the sample size of trajectories at a given starting distance.

Other variants are possible. For instance, in step 1, when the starting distances of the data are discrete, one may not need to subdivide the data by a range of starting distances but could instead obtain a median from each distance. In step 3, instead of using the inverse slope, an alternative way to measure deviation from perfect plasticity is by comparing angles of the empirically generated best-fit lines. The range of starting distances for the best-fit line may be limited to the physically maximum starting distance or the maximum relevant starting distance along the $x$ axis. For instance, if the goal is a food source at the center of petri dish and the goal directed entities are the bacteria swimming up the food molecule gradient, the physical maximum starting distance will be the radius of the petri dish, or the radius of the concentration gradient that has biologically meaningful concentration.

\section{Data Filtering for Demonstration 1}

Micali et al. (2017) set up a fixed, linear gradient of MeAsp, a non-metabolizable chemoattractant, in a microfluidics device. Escerichia coli are released in the device, able to move freely (Figure 3). After the gradient becomes stable, bacterial motion data are collected 3 times at 1-hour interval for each of 3 repeats (total of 9 repeats). The motions of $E$. coli were digitized in a coordinate system where the MeAsp gradient extends along the $x$ axis from 0 to 1022 . In this coordinate system, an $x$ coordinate of 0 is the highest concentration and 1022, the lowest. The $x$ and $y$ coordinate of $E$. coli were recorded every frame ( 1 second $=30$ frames), but only the $x$ coordinate was used for our analysis, in effect making the data one dimensional.

The raw data for $E$. coli chemotaxis, i.e., the $x$ coordinates for $E$. coli in the channel, was filtered for the demonstration. Among the trajectories that lasted for at least 2 seconds, the $x$ coordinates were collected for each trajectory every 0.5 seconds. Then, the consecutive $x$ coordinates of each trajectory were compared to decide whether the bacteria moved toward higher concentration, which was treated as the direction toward the goal. In other words, a bacterium's change in $x$ coordinates after 0.5 seconds was used to determine whether the move 


\section{Ratio of good moves across MeAsp gradient}

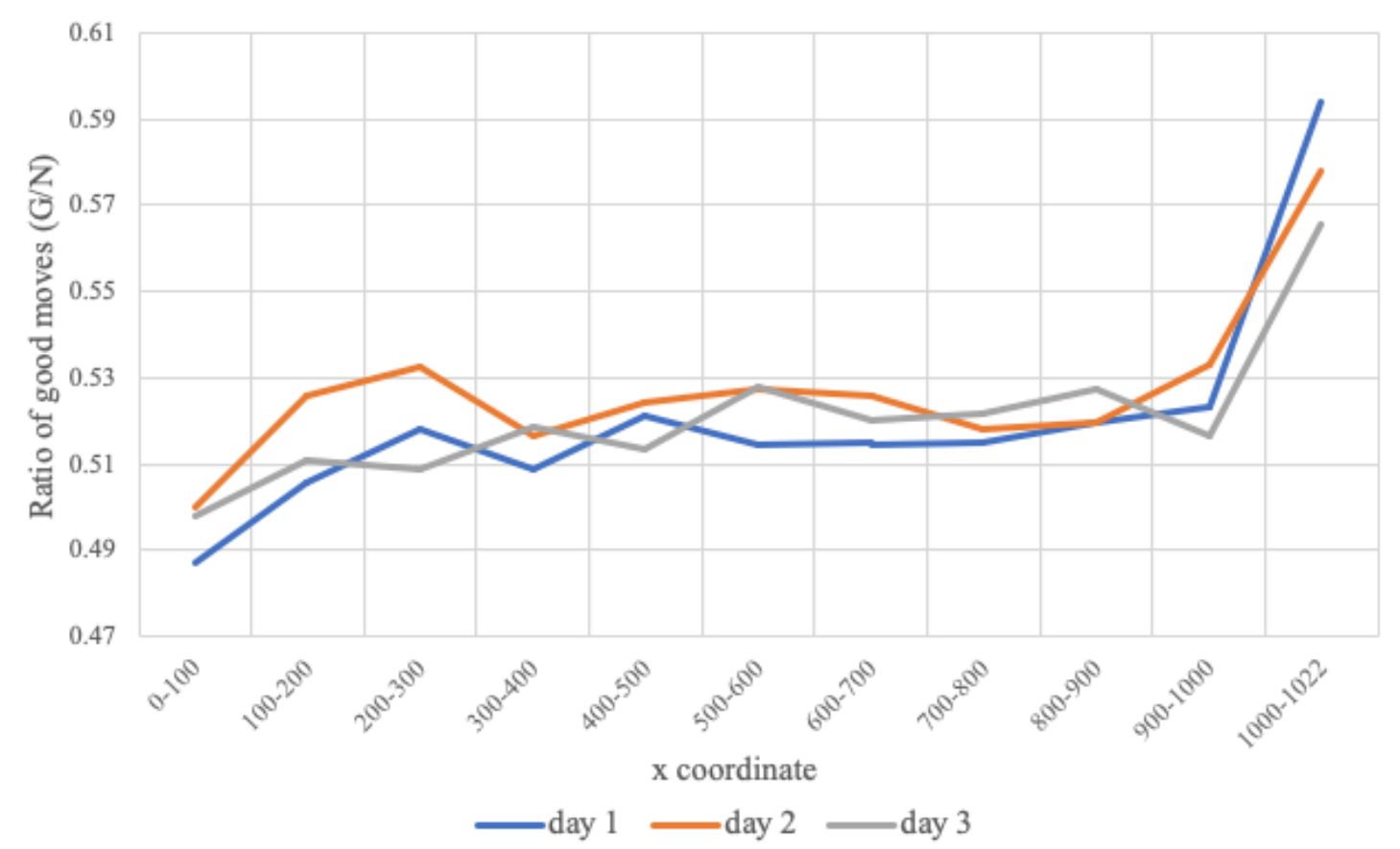

Figure S1: Ratio of good moves along the MeAsp gradient on different days. We did not detect significant difference in the ratio of good moves or other patterns along the $x$ coordinate on different days despite approximately $10 \%$ concentration difference between day 3 and the other days. Thus, we combined the chemotaxis data of all 9 repeats to generate persistence values for each band.

was "good" (moved to smaller $x$ coordinates after 0.5 seconds) or "bad" (moved to greater $x$ coordinate). The $x$ axis was subdivided into a series of bands that have the same width to ensure that only moves occurring under relatively similar environmental conditions were combined to produce a single persistence value (Figure 3). The $x$ axis from 0 to 1022 was divided into 10 bands, each with width of 100 , and 1 band with width of 22 . All the moves that start from an $x$ coordinate within a band were grouped together to generate the persistence measure of that specific band. The final statistics for persistence represented in the figures and the table below were obtained by merging the data for all 9 repeats.

For the three days of experiments, there was approximately $10 \%$ difference in concentration gradient of MeAsp at a given band. Between $x$ coordinates of 0 (highest concentration) and 1022 (lowest concentration), the relative gradient of MeAsp with respect to the chamber $(200 \mu \mathrm{M})$ ranged from 0.43251 to 0.60446 on day 1 ; from 0.42162 to 0.60570 on day 2 ; from 0.47855 to 0.66483 on day 3 . We assumed this difference in concentration gradient to be negligible because we did not detect significant difference in ratio of good moves or patterns across $x$ coordinate over different days (Figure $\mathrm{S} 1$ ). 
Table S1: Ratio of good moves and persistence on different days

\begin{tabular}{lccccccrrr}
\hline & \multicolumn{2}{c}{ Day 1 } & \multicolumn{2}{c}{ Day 2 } & \multicolumn{2}{c}{ Day 3 } & \multicolumn{2}{c}{ Combined } \\
\hline Band & G/N & \multicolumn{1}{c}{$\mathrm{P}$} & G/N & $\mathrm{P}$ & \multicolumn{1}{c}{ G/N } & \multicolumn{1}{c}{$\mathrm{P}$} & \multicolumn{1}{c}{ G/N } & \multicolumn{1}{c}{$\mathrm{P}$} \\
\hline $0-100$ & 0.4870 & -0.0260 & 0.5001 & 0.0003 & 0.4981 & -0.0038 & 0.4959 & -0.0082 \\
$100-200$ & 0.5057 & 0.0113 & 0.5261 & 0.0521 & 0.5108 & 0.0216 & 0.5164 & 0.0328 \\
$200-300$ & 0.5179 & 0.0357 & 0.5324 & 0.0648 & 0.5086 & 0.0172 & 0.5219 & 0.0438 \\
$300-400$ & 0.5089 & 0.0178 & 0.5165 & 0.0331 & 0.5186 & 0.0372 & 0.5147 & 0.0294 \\
$400-500$ & 0.5210 & 0.0421 & 0.5241 & 0.0481 & 0.5136 & 0.0271 & 0.5199 & 0.0398 \\
$500-600$ & 0.5145 & 0.0291 & 0.5275 & 0.0551 & 0.5276 & 0.0553 & 0.5227 & 0.0454 \\
$600-700$ & 0.5151 & 0.0302 & 0.5257 & 0.0513 & 0.5200 & 0.0401 & 0.5202 & 0.0405 \\
$700-800$ & 0.5152 & 0.0304 & 0.5182 & 0.0365 & 0.5215 & 0.0431 & 0.5182 & 0.0363 \\
$800-900$ & 0.5196 & 0.0392 & 0.5196 & 0.0391 & 0.5272 & 0.0543 & 0.5216 & 0.0432 \\
$900-1000$ & 0.5232 & 0.0465 & 0.5329 & 0.0658 & 0.5165 & 0.0330 & 0.5259 & 0.0517 \\
$1000-1022$ & 0.5941 & 0.1882 & 0.5779 & 0.1557 & 0.5656 & 0.1312 & 0.5786 & 0.1571 \\
\hline
\end{tabular}

4. Summary of Persistence Analysis for Demonstrations 1 and 2

Table S2: Summary of the results from Demonstration 1

\begin{tabular}{lrrllr}
\hline Band & Good $(\mathrm{G})$ & Total $(\mathrm{N})$ & G/N & $\mathrm{R}$ & Persistence \\
\hline $0-100$ & 12884 & 25982 & 0.49588 & 0.50000 & -0.00824 \\
$100-200$ & 14355 & 27799 & 0.51639 & 0.50000 & 0.03277 \\
$200-300$ & 14510 & 27803 & 0.52189 & 0.50000 & 0.04377 \\
$300-400$ & 13283 & 25808 & 0.51469 & 0.50000 & 0.02937 \\
$400-500$ & 14329 & 27560 & 0.51992 & 0.50000 & 0.03984 \\
$500-600$ & 13748 & 26303 & 0.52268 & 0.50000 & 0.04536 \\
$600-700$ & 13309 & 25583 & 0.52023 & 0.50000 & 0.04046 \\
$700-800$ & 12885 & 24867 & 0.51816 & 0.50000 & 0.03631 \\
$800-900$ & 12478 & 23923 & 0.52159 & 0.50000 & 0.04318 \\
$900-1000$ & 12927 & 24582 & 0.52587 & 0.50000 & 0.05175 \\
$1000-1022$ & 3045 & 5263 & 0.57857 & 0.50000 & 0.15713 \\
\hline
\end{tabular}

Table S3: Summary of the results from Demonstration 2

\begin{tabular}{lrrrlcr}
\hline Distance & Good $(\mathrm{G})$ & Bad & Total $(\mathrm{N})$ & $\mathrm{G} / \mathrm{N}$ & $\mathrm{R}$ & Persistence \\
\hline$[1,2)$ & 89 & 47 & 136 & 0.65441 & 0.37929 & 0.44324 \\
{$[2,3)$} & 101 & 53 & 154 & 0.65584 & 0.43561 & 0.39022 \\
{$[3,4)$} & 85 & 35 & 120 & 0.70833 & 0.44167 & 0.47761 \\
{$[4,5)$} & 54 & 38 & 92 & 0.58696 & 0.45833 & 0.23746 \\
{$[5,6)$} & 72 & 60 & 132 & 0.54545 & 0.46591 & 0.14894 \\
{$[6,7)$} & 40 & 28 & 68 & 0.58824 & 0.46569 & 0.22936 \\
{$[7,8)$} & 32 & 21 & 53 & 0.60377 & 0.46855 & 0.25444 \\
{$[8,9)$} & 19 & 19 & 38 & 0.50000 & 0.45175 & 0.08800 \\
& & & & & \multicolumn{2}{c}{ ○ OPEN ACCESS- PTPBIO.ORG }
\end{tabular}




\begin{tabular}{lrrrllr}
\hline Distance & Good $(\mathrm{G})$ & Bad & Total $(\mathrm{N})$ & G/N & $\mathrm{R}$ & Persistence \\
\hline$[9,10)$ & 18 & 16 & 34 & 0.52941 & 0.46814 & 0.11521 \\
{$[10,11)$} & 19 & 12 & 31 & 0.61290 & 0.48118 & 0.25389 \\
{$[11,12)$} & 13 & 8 & 21 & 0.61905 & 0.48413 & 0.26154 \\
{$[12,13)$} & 4 & 4 & 8 & 0.50000 & 0.52083 & -0.04348 \\
{$[13,14)$} & 4 & 6 & 10 & 0.40000 & 0.51667 & -0.24138 \\
{$[14,15)$} & 8 & 3 & 11 & 0.72727 & 0.49242 & 0.46269 \\
{$[15,16)$} & 6 & 1 & 7 & 0.85714 & 0.50000 & 0.71429 \\
{$[16,17)$} & 2 & 0 & 2 & 1.00000 & 0.50000 & 1.00000 \\
\hline
\end{tabular}

\section{Acknowledgments}

We thank Dr. John Mercer for his thoughtful comments on an earlier version of this paper, and two reviewers for comments on later versions. The bacteria data were generously provided by Dr. Remy Colin and Dr. Victor Sourjik at the Max Planck Institute for Terrestrial Microbiology. We would also like to acknowledge Inchan Hwang and James Cho, who provided substantial computational support and critical insights in developing ideas for this project.

\section{Literature cited}

Atkin, O. K., D. Bruhn, and M. G. Tjoelker. 2005. "Response of Plant Respiration to Changes in Temperature: Mechanisms and Consequences of Variations in Q10 Values and Acclimation." In Plant Respiration, edited by H. Lambers and M. Ribas-Carbó, 95-135. Dordrecht: Springer.

Bailey, H. and P. Thompson. 2006. "Quantitative Analysis of Bottlenose Dolphin Movement Patterns and Their Relationship With Foraging." Journal of Animal Ecology 75 (2): 456-65.

Bailey, J. D., J. Wallis, and E. A. Codling. 2018. "Navigational Efficiency in a Biased and Correlated Random Walk Model of Individual Animal Movement.” Ecology 99 (1): 217-223.

Batschelet, E. 1981. Circular Statistics in Biology. New York: Academic Press.

Bearon, R. N. and W. M. Durham. 2019. "A Model of Strongly Biased Chemotaxis Reveals the Trade-Offs of Different Bacterial Migration Strategies.” Mathematical Medicine and Biology dqz007: 1-34.

Beauvais, F., L. Michel, and L. Dubertret. 1995. "Exogenous Nitric Oxide Elicits Chemotaxis of Neutrophils in Vitro." Journal of Cellular Physiology 165 (3): 610-614.

Benhamou, S. 2004. "How to Reliably Estimate the Tortuosity of an Animal'S Path: Straightness, Sinuosity, or Fractal Dimension?” Journal of Theoretical Biology, 229 (2): 209-220.

Benhamou, S. 2006. "Detecting an Orientation Component in Animal Paths When the Preferred Direction Is Individual-Dependent.” Ecology 87 (2): 518-528.

Benhamou, S., F. Bonadonna, and P. Jouventin 2003. "Successful Homing of Magnet-Carrying White-Chinned Petrels Released in the Open Sea." Animal Behaviour 65 (4): 729-734.

Benhamou, S. and P. Bovet. 1992. "Distinguishing Between Elementary Orientation Mechanisms by Means of Path Analysis.” Animal Behaviour 43 (3): 371-377. doi:10.1016/S00033472(05)80097-1.

Berg, H. C. 1993. Random Walks in Biology. Princeton: Princeton University Press. 
Bovet, P. and S. Benhamou. 1988. "Spatial Analysis of Animals' Movements Using a Correlated Random Walk Model." Journal of Theoretical Biology 131 (4): 419-33.

Braithwaite, R. B. 1953. Scientific Explanation. Cambridge: Cambridge University Press.

Codling, E. A., M. J. Plank, and S. Benhamou. 2008. "Random Walk Models in Biology.” Journal of the Royal Society Interface 5 (25): 813-834.

Doyle, P. G., and J. L. Snell. 1984. Random Walks and Electric Networks. Washington, DC: Mathematical Association of America.

Ehring, D. 1984. “The System-Property Theory of Goal-Directed Processes." Philosophy of the Social Sciences 14 (4): 497-504.

Fortin, D., J. M. Morales, and M. S. Boyce. 2005. "Elk Winter Foraging at Fine Scale in Yellowstone National Park." Oecologia 145: 335-343.

Gardner, H. 1983. Frames of Mind: The Theory of Multiple Intelligences. New York: Basic Books.

Garson, J. A. 2016. A Critical Overview of Biological Functions. Dordrecht: Springer International Publishing.

Gershenson, C., and N. Fernández. 2012. "Complexity and Information: Measuring Emergence, SelfOrganization, and Homeostasis at Multiple Scales." Complexity 18 (2): 29-44.

Hill, R. 1950. The Mathematical Theory of Plasticity. London: Oxford University Press.

Hill, N. A., D. P. Häder. 1997. "A Biased Random Walk Model for the Trajectories of Swimming Microorganisms." Journal of Theoretical Biology 186: 503-526.

Kareiva, P. M. and N. Shigesada. 1983. "Analyzing Insect Movement as a Correlated Random Walk.” Oecologia 56 (2-3), 234-238.

Khaldy, L., O. Peleg, C. Tocco, L. Mahadevan, M. Byrne, and M. Dacke, 2019. "The Effect of Step Size on Straight-Line Orientation.” Journal of the Royal Society Interface 16 (157): 20190181.

Lind, M., B. Deleuran, K. Thestrup, Pedersen, K. Søballe, E. F. Eriksen, and C. Bünger. 1995. "Chemotaxis of Human Osteoblasts." APMIS 103: 140-146.

Marsh, L. M., and R. E. Jones. 1988. "The Form and Consequences of Random Walk Movement Models." Journal of Theoretical Biology 133: 113-131.

Mayr, E. 1974. "Teleological and Teleonomic, a New Analysis." In Methodological and Historical Essays in the Natural and Social Sciences, edited by R. S. Cohen and M. W. Wartofsky, 91-117. Dordrecht: Springer.

McClintock, B. T., R. King, L. Thomas, J. Matthiopoulos, B.J ., McConnell, and J. M. Morales. 2012. "A General Discrete-Time Modeling Framework for Animal Movement Using Multistate Random Walks.” Ecological Monographs 82 (3), 335-349.

McCutcheon, M. 1946. “Chemotaxis in Leukocytes.” Physiological Reviews 26 (3): 319-336.

McShea, D. W. 2012. "Upper-Directed Systems: A New Approach to Teleology in Biology.” Biology E Philosophy 27 (5): 663-684.

McShea, D.W. 2016a. "Hierarchy: The Source of Teleology in Evolution." In Evolutionary Theory: A Hierarchical Perspective, edited by Niles Eldredge, Telmo Pievani, Emanuele Serrelli, and Ilya Tëmkin, 86-102. Chicago: University of Chicago Press.

McShea, D.W. 2016b. "Freedom and Purpose in Biology." Studies in History and Philosophy of Biological and Biomedical Sciences 58: 64-72.

Micali, G., R. Colin, V. Sourjik, and R. G. Endres. 2017. "Drift and Behavior of E. coli Cells." Biophysical Journal 113 (11): 2321-2325.

๑ OPEN ACCESS - PTPBIO.ORG 
Nagel, E. 1979. Teleology Revisited, and Other Essays in the Philosophy and History of Science. New York: Columbia University Press.

Nathan, R., W. M. Getz, E. Revilla, M. Holyoak, R. Kadmon, D. Saltz, and P. E. Smouse. 2008. “A Movement Ecology Paradigm for Unifying Organismal Movement Research." Proceedings of the National Academy of Sciences, 105 (49): 19052-19059.

Orr, W., and P. A. Ward. 1978. "Quantitation of Leukotaxis in Agarose by Three Different Methods.” Journal of Immunological Methods 20: 95-107.

Pahl, M., H. Zhu, J. Tautz and S. Zhang. 2011. "Large Scale Homing in Honeybees.” PLoS One 6 (5), e19669.

Papastamatiou, Y. P., D. P. Cartamil, C. G. Lowe, C. G. Meyer, B. M. Wetherbee, and K. N. Holland. 2011. "Scales of Orientation, Directed Walks and Movement Path Structure in Sharks." Journal of Animal Ecology 80 (4): 864-874.

Patlak, C. S. 1953. "Random Walk With Persistence and External Bias." The Bulletin of Mathematical Biophysics 15 (3): 311-338.

Peleg, O., and L. Mahadevan. 2016. "Optimal Switching Between Geocentric and Egocentric Strategies in Navigation.” Royal Society Open Science 3: 7.

Qasaimeh, M. A., M. Pyzik, M. Astolfi, S. M. Vidal, and D. Juncker. 2018. "Neutrophil Chemotaxis in Moving Gradients." Advanced Biosystems 2 (7): 1700243.

Ramırez-Gómez, H. V., I. Tuval, A. Guerrero, and A. Darszon. 2019. "Analysis of Sperm Chemotaxis." Methods in Cell Biology 151: 473-486.

Rosenblueth, A., Wiener, N., and Bigelow, J. 1943. "Behavior, Purpose and Teleology." Philosophy of Science 10 (1): 18-24.

Scheffler, I. 1959. "Thoughts on Teleology." The British Journal for the Philosophy of Science 9 (36): 265284.

Schultz, C. B., and E. E. Crone. 2001. "Edge-Mediated Dispersal Behaviour in a Prairie Butterfly." Ecology 82: 1879-1892.

Sommerhoff, G. 1950. Analytical Biology. London: Oxford University Press.

Tanaka, Y., M. Jahan, G. Sarowar, T. Kondo, M. Nakano, and S. Yumura. 2019. "Cytokinesis D Is Mediated by Cortical Flow of Dividing Cells Instead of Chemotaxis." Cells 8 (5): 473.

Turchin, P. 1996. "Fractal Analyses of Animal Movement: A Critique." Ecology 77 (7): 2086-2090.

Turchin, P. 1998. Quantitative Analysis of Movement: Measuring and Modeling Population Redistribution in Animals and Plants. Sunderland, MA: Sinauer Associates.

Varennes, J., H. Moon, S. Saha, A. Mugler, and B. Han. 2019. "Physical Constraints on Accuracy and Persistence During Breast Cancer Cell Chemotaxis." PLoS Computational Biology 15 (4): e1006961.

Weimerskirch, H., F. Bonadonna, F. Bailleul, G. Mabille, G. Dell'Omo, and H.-P. Lipp. 2002. "GPS Tracking of Foraging Albatrosses." Science 295 (5558): 1259-1259.

West-Eberhard, M. J. 2003. Developmental Plasticity and Evolution. Oxford: Oxford University Press.

Woodfield, A. 1976. Teleology. Cambridge: Cambridge University Press.

(C) 2020 Author(s). This is an open-access article distributed under the terms of the Creative Commons Attribution 4.0 International license, which permits anyone to download, copy, distribute, display, or adapt the text without asking for permission, provided that the creator(s) are given full credit. 